\title{
Three-dimensional magnetic reconnection and its application to solar flares
}

\author{
Miho Janvier $\dagger$ \\ Institut d'Astrophysique Spatiale, CNRS, Univ. Paris-Sud, Université Paris-Saclay, \\ Bât. 121, 91405 Orsay CEDEX, France
}

(Received 14 October 2016; revised 25 December 2016; accepted 4 January 2017)

Solar flares are powerful radiations occurring in the Sun's atmosphere. They are powered by magnetic reconnection, a phenomenon that can convert magnetic energy into other forms of energy such as heat and kinetic energy, and which is believed to be ubiquitous in the universe. With the ever increasing spatial and temporal resolutions of solar observations, as well as numerical simulations benefiting from increasing computer power, we can now probe into the nature and the characteristics of magnetic reconnection in three dimensions to better understand the phenomenon's consequences during eruptive flares in our star's atmosphere. We review in the following the efforts made on different fronts to approach the problem of magnetic reconnection. In particular, we will see how understanding the magnetic topology in three dimensions helps in locating the most probable regions for reconnection to occur, how the current layer evolves in three dimensions and how reconnection leads to the formation of flux ropes, plasmoids and flaring loops.

Key words: astrophysical plasmas, plasma simulation, space plasma physics

\section{Introduction}

Solar flares are the most energetic events taking place in our solar system. They are detected as a sudden increase in the X-ray light emission as they take place in our Sun's atmosphere. Their energy ranges from $10^{24} \mathrm{erg}$ (the detection lower limit) to $10^{32} \mathrm{erg}$ (Schrijver et al. 2012), or in Joules $10^{17}-10^{25} \mathrm{~J}$. They can then be classified or ranked with the intensity of the light curve peak in soft X-rays, as recorded by the Geostationary Operational Environmental Satellites (GOESs) near Earth. Their emissions are recorded in a wide range of the electromagnetic spectrum, from gamma-rays and X-rays to radio wavelengths. With a varied range of instruments aboard spacecraft and on the ground, our Sun can be studied in an incredible amount of detail.

While less powerful flares (A, B and C-class flares) are most often confined flares (their influence on the corona remains localised), other flares of higher intensities (so-called M- and X-class flares) can be responsible for the release of large clouds of solar plasma - called coronal mass ejections (CMEs) (e.g. Yashiro et al. 2006) - in the interplanetary medium. These are also detected in the interplanetary medium (then

$†$ Email address for correspondence: miho.janvier@ias.u-psud.fr 
referred to as interplanetary CMEs). They perturb the ambient solar wind and have characteristic signatures in terms of magnetic field, proton temperature, composition and ionisation ratio different than the ambient medium. Relativistic solar energetic particles, accelerated from the Sun during flares, as well as CMEs, are important drivers of space weather, since they impact the magnetic environments of planets (see Gosling et al. 1991; Prangé et al. 2004). Then, understanding the underlying mechanisms of these eruptive flares is of primary importance to better assess the likelihood and the evolution of CMEs in the interplanetary medium. Understanding the flaring mechanism also questions the habitability of exoplanets orbiting around other stars.

Since most of the energy available in the Sun's corona is in magnetic form (low- $\beta$ plasma), and as large solar flares mainly occur in the locations of sunspots, it is natural to suppose that solar flares are powered by magnetic energy. Then, there is a need for a mechanism that can convert this magnetic energy into other forms of energy such as heat, particle energies, etc. Such work started with Cowling and Sweet (among others, see e.g. Cowling 1945; Sweet 1950) who investigated the separation of the motion of the magnetic field and the plasma. Later, Dungey (Dungey 1953) continued this work, proposing the existence of a surface where a strong ohmic electric field exists due to the decrease of the conductivity of the medium, thus allowing changes in the 'identity' of field lines. This change of identity can be illustrated by magnetic field lines changing their connection with one another, hence the term magnetic reconnection.

Several years later, Sweet (Sweet 1956) and Parker (Parker 1957b) proposed the formation of a current layer near magnetic nulls, where the magnetic field can be dissipated (see also $\$ 2$ ). Such a mechanism was put forward to explain the acceleration of solar particles to relativistic speeds (Parker 1957a; Sweet 1958b). Indeed, although it is impossible to directly probe the Sun's atmosphere to measure the magnetic field, consequences of magnetic reconnection were first observed as populations of energetic particles (solar cosmic rays) and radio bursts (as summarised in e.g. Wild, Smerd \& Weiss 1963). Theoretical models continue to be confronted with observations. For example, intensely emitting magnetic arcades loops and loop tops seen during flares (e.g. Masuda et al. 1994), coronal mass ejections (Webb \& Howard 2012) and plasmoids (which can be defined as a circular plasma structure seen on a projection on the plane of the sky, e.g. Takasao et al. 2012) are indicative of magnetic structures that are similar to those found in simulations. Moreover, inflows and flare ribbons sweeping the Sun's surface (see references in McKenzie 2011) are also believed to be indirect observable consequences of reconnecting magnetic fields.

Then, since Sweet's and Parker's seminal works in laying the basis for a magnetic reconnection model, scenarios explaining how this phenomenon actually takes place have flourished. First, we can distinguish between models that are focussed on the process of reconnection itself. These models, with consideration of topology and/or energetics, can have a more or less complete description of the Ohm's law (including the Hall term and/or other kinetic effects at ion or electron scales, see e.g. Birn et al. 2001), and provide analyses of the evolution of the dissipation layer, which can become unstable (e.g. the tearing instability) or into which the magnetic field can be forced to flow and reconnect. Over the years, many papers have been dedicated to the understanding of these fundamental processes at various scales, and such studies are extremely valuable to better assess the energetics of observed flares. Dedicated plasma experiments (such as MRX, Yamada et al. 1997) and recent space missions (e.g. CLUSTER and MMS, Sergeev et al. 2008; Fuselier et al. 2016) also shed 
some light on the evolution of the magnetic field, plasma and non-thermal particle populations during reconnection events. As will be seen in the following, magnetic reconnection remains a highly investigated domain due to its complexity. Because of the multiple approaches and the different highlights (kinetic effects, topology, energy, particles, ...), a review covering all these aspects is out the scope of the present work. We propose instead to the reader the reviews of Yamada (2007), Zweibel \& Yamada (2009) and Yamada, Kulsrud \& Ji (2010) for more information on aspects of reconnection at the diffusion region scale, in the magnetosphere and in laboratory experiments.

With the first observations of flares (see the well-documented early report by Severny 1964b), several models started to emerge from the middle of the 20th century (see Sweet 1969, and references therein). Of particular interest here are global models which aid in understanding the global evolution of the magnetic field and generic consequences that are observable. These models were initially two-dimensional (for example, the so-called CSHKP model, see Carmichael 1964; Sturrock 1966; Hirayama 1974; Kopp \& Pneuman 1976). They evolved along the years to include more and more physical aspects of flares (heating of loops, energetic particles, etc.), as observations were made with better temporal and spatial resolutions. In this two-dimensional (2-D) model, the flare is powered by magnetic reconnection at a vertical current sheet forming in the corona below the ejected large-scale magnetic structure (or upward-propagating plasmoid, where here the plasmoid refers to a region where the magnetic field takes a circular shape, in a 2-D projection) that forms the CME. Particles accelerated from, or waves generated at, the reconnection region, travel along field lines and impact/heat the chromosphere, the lowest and densest layer of the Sun's atmosphere. Localised heating is then seen as flare ribbons, that are believed to form at the footpoints of newly reconnected field lines, or coronal loops, which themselves are heated to extremely high temperatures (they can be seen in soft X-rays) and filled with dense plasma upon 'evaporation' from the chromosphere.

However, since the nature of flares is intrinsically three-dimensional, those models are not able to completely describe certain features of flares. For example, a selfconsistent model describing the evolution of the ejected magnetic structure, from its formation to its further expansion as well as its flux increase, is still lacking. Note that the ejected structure is often modelled as a flux rope, which can be described as a bundle of magnetic field lines twisting around each other. Furthermore, the evolution of flare loops displays a gradual transition from strongly sheared flare loop arcades to a nearly potential configuration (e.g. Asai et al. 2003; Su et al. 2006, 2007; Warren, O'Brien \& Sheeley 2011), while the morphology of flare ribbons is also peculiar (with a $J$-shape, see figure 6 in Chandra et al. 2009). Most importantly, the nature of reconnection in three dimensions is also not addressed in the CSHKP model and similar models. Over the past few years, 3-D numerical simulations have helped our understanding of the evolution of an unstable flux-rope/magnetic configuration (e.g. Aulanier et al. 2010; Aulanier, Janvier \& Schmieder 2012; Kusano et al. 2012), the evolution of the current layer and reconnection in three dimensions (Janvier et al. 2013; Kliem et al. 2013) as well as its consequences for interpreting solar flares (e.g. Dudík et al. 2014).

In the following, we review certain aspects of magnetic reconnection applied to the understanding of eruptive flares. We are aware that related reviews already exist (e.g. Pontin 2012), although generally these are focused on the analytical/simulation side or observational side. With the recent advances in understanding reconnection 
in three dimension and the growing evidence of observational data from solar flares confirming evidence laid by theoretical works, we thought it beneficial to review their parallel evolution in the following. We also place them in a general context of reconnection studied in different fields of plasma studies. As a first step, we will see in $\S 2$ how the magnetic topology allows one to investigate the regions were magnetic reconnection is most likely to occur, such as null points, separatrices, or more generally, quasi-separatrix layers, which generalise the concepts of separatrices in three dimensions. We will also investigate the different diffusion mechanisms that can take place in the current layer in $\S 3$ (e.g. the onset of instabilities), as well as the current layer formation, evolution and observation in eruptive flares. The consequences of reconnection on the magnetic field (slipping of field lines, formation of flare loops, flux ropes and plasmoids) are investigated in $\S 4$. Finally, in $\S 5$, we summarise our results, discuss the limitations of present studies of reconnection applied to solar flares and conclude.

\section{Magnetic topology of solar flares}

Solar flares are believed to be powered by a physical phenomenon called magnetic reconnection. When reconnection occurs, magnetic field lines rearrange their connectivity so as to create new magnetic structures. Then, two questions arise: what is this region where reconnection takes place? And where does it form?

\subsection{Null points, separatrices and separators}

In the 1950s, the idea emerged that some plasma locations can be related with the magnetic field losing its identity: in other words, regions where the magnetic field decorrelates from the plasma. Sweet and Parker (as summarised in Parker 1957b) provided a mechanism for the spontaneous formation of a diffusion layer, even in highly conducting media. Magnetic fields with opposite directions, when pressed together by external forces, form a surface where the electric current density is large (figure $1 b$ ). Subsequently, the gradient in the field density becomes so important that the diffusion terms, generally of negligible importance away from this singular layer, are responsible for the rapid diffusion of the magnetic field (see $\S 3.1$ for a more detailed description of the different dissipation processes).

Sweet's and Parker's proposal of tangential discontinuities was soon after extended by Sweet (Sweet 1958a) to the presence of magnetic nulls in a volume (following the concept of Giovanelli 1947), where the magnetic field vanishes. Null points were seen as physically interesting in the concept of flares, as they were thought to offer the best locations for particle acceleration (as these locations would be associated with a parallel electric field). Sweet proposed that null points result from flux tubes protruding from sunspots. The distorted magnetic field in their vicinity then allows magnetic reconnection to occur. This work, along with that of Sweet (1958c), was important in laying the basis of the study of the topology of the magnetic field and the exchange of flux between different magnetic connectivity domains traced by the lines of magnetic force. As null points are locations where the magnetic field line mapping is discontinuous, ideal kinematic solutions lead to a region where the magnetic field must be rapidly dissipated: this concept of current layers around null points was introduced by Syrovatskiǐ (1971).

Magnetic null points are associated with current layers, i.e. regions where the electric current density increases, either from a spontaneous formation due to instabilities $(\$ 3)$ or from a dynamic formation as a result of the magnetic field evolution. Analyses of the flow pattern near the null point reveal some geometrical 
(a)

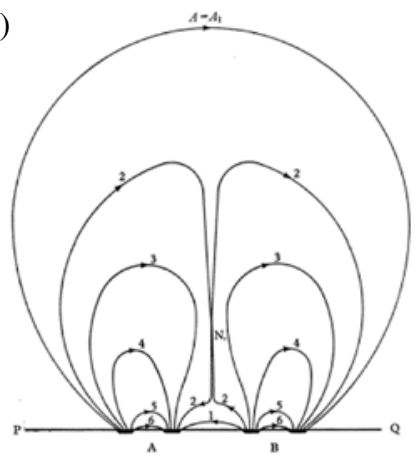

(b)

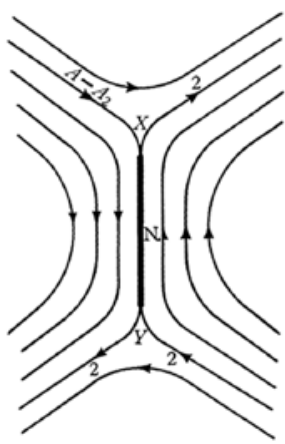

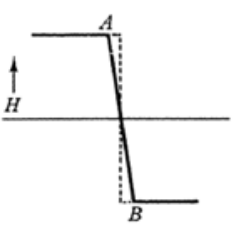

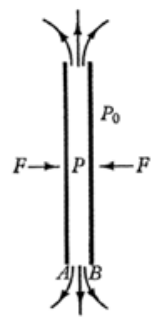

FIGURE 1. Early analytical models of reconnection regions: (a) Sweet's mechanism at play when two bipolar sunspots are brought close to each other forming a current layer surrounding the null point N1. (b) Sweet and Parker collision layer (current sheet), with a description of the magnetic field (here called $\mathrm{H}$ ), and the hydrodynamic model $(a)$. Adapted from Sweet (1958a).

properties of the current layer such as its length in quasistatic field evolution (see e.g. Syrovatskii 1966; Priest \& Raadu 1975). Then, the consequences of magnetic reconnection in those singular regions were investigated analytically in more detail, although the first attempts mainly treated the problem as a boundary layer associated with some singular structure in a nearly ideal plasma (e.g. Sonnerup 1970; Yeh \& Axford 1970).

Studies of kinematic reconnection at null points were soon extended from two dimensions to three dimensions (e.g. Yeh 1976; Baum et al. 1979; Lau \& Finn 1990). This led to a definition of various topological objects, such as separators, which connect pairs of null points of opposite sign. The sign is defined with the highest number of positive or negative eigenvalues: since $\boldsymbol{\nabla} \cdot \boldsymbol{B}=0$, two eigenvalues are positive and one is negative, or the reverse. In the presence of a separator, the current layer tends to form along it. Then, separatrices are lines (or surfaces in three dimensions) separating different domains of connectivity. Note that separatrices are also locations of current sheet formation when the magnetic field is sheared (e.g. Vekstein, Priest \& Amari 1991). Properties of nulls can also be found in Lau \& Finn (1990), who described the three eigenvalues and eigenvectors of the magnetic field near the null point. Then, there is a specific field line that passes through the null, called the spine, which is associated with the eigenvalue with the single sign. All the other field lines connecting the null form a surface called the fan, associated with the two eigenvalues of the same sign (see figure 2 and Priest \& Titov 1996). Overall, topological connectivity was regarded as essential as it contains information regarding the location and the structure where localised electric currents form during an evolution of the magnetic configuration.

Separatrices and separators, similar to null points, are regions of discontinuous field line mapping. Their role in the formation of discontinuities in the plasma, for example in the electric field, can be investigated by ideal kinematic solutions. Over the years, different regimes of reconnection have been studied, from a kinematic approach to a dynamic approach with the help of numerical simulations. Depending on whether the process takes place at null points (see Parnell et al. 1996; Priest \& Titov 1996; Pontin, Hornig \& Priest 2004, 2005; Pontin, Priest \& Galsgaard 2013, and references therein for more explanation on the fan, spine reconnection 


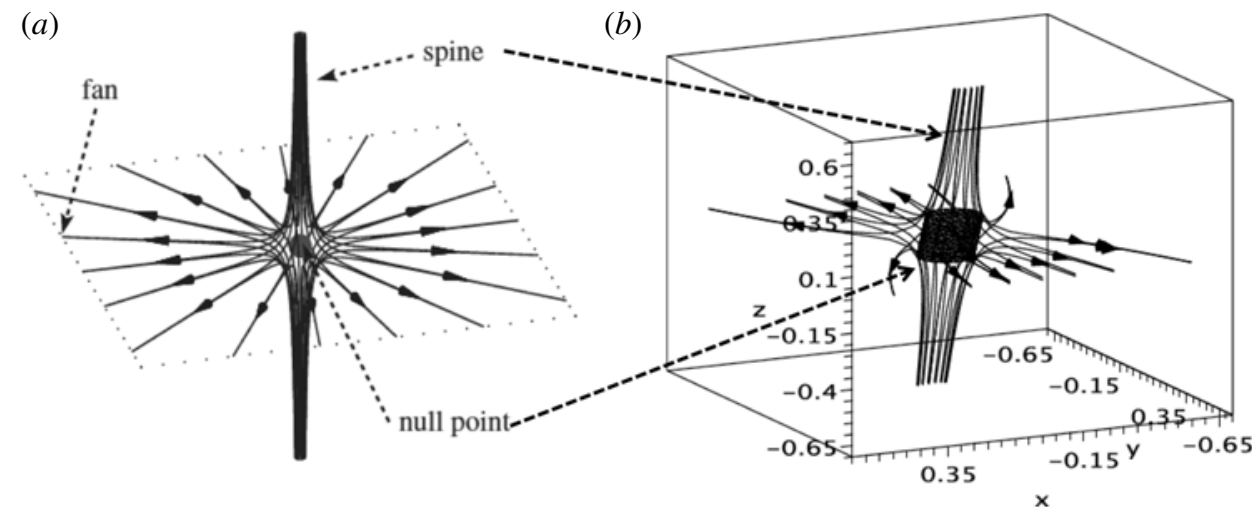

FIGURE 2. Different topological definitions in the presence of a null point: specific field lines that pass through the null form the spine, then they spread in the fan plane (defined by the two eigenvectors with same sign eigenvalues). (a) represents a symmetric case, associated with two equal eigenvalues in the fan plane (Pontin 2012), while (b) shows an asymmetric null point (adapted from Al-Hachami \& Pontin (2010)). In the present plots, the field lines are selected to pass nearby the spine and the fan.

regimes), or at separators, i.e. with currents concentrated along a separator field line (Longcope \& Cowley 1996; Heerikhuisen \& Craig 2004; Pontin \& Craig 2006; Parnell, Haynes \& Galsgaard 2010; Stevenson \& Parnell 2015). The papers of Priest \& Pontin (2009) and Priest (2016) provide an overview of the different reconnection regimes happening around the null point as well as a link to different works on this topic. They also provided an extension to the classical definition of reconnection at spine/fan locations by defining three regimes: torsional spine, torsional fan and spine fan reconnection. The former two occur when rotational motions (of either the spine or the fan) lead to the concentration of current along the spine or the fan, while the latter occurs when the current is concentrated along both fan and spine due to the shearing of a null point. Although the early works were kinematic studies of reconnection around null points, over the years computer studies have made possible dynamic studies of reconnection and evolution of the current sheet near a null point (e.g. Pontin, Bhattacharjee \& Galsgaard 2007; Galsgaard \& Pontin 2011), comparison with observations (e.g. Mandrini et al. 1991; Masson et al. 2009) as well as investigation beyond the classical magneto-hydrodynamic (MHD) frame (e.g. in collisionless plasmas, Tsiklauri \& Haruki 2007).

Dynamics surrounding a null point can also be investigated in laboratory experiments. In Syrovatskii, Frank \& Khodzhaev (1972), Baum et al. (1973), Baum \& Bratenahl (1976), the authors showed that the plasma resistivity indeed becomes anomalously large, and the current becomes concentrated near null points. Recent laboratory experiments such as in Stenzel et al. (2002) teach us that the small-scale physics involving ion and electron dynamics must also be carefully considered in the surrounding of null points. Finally, in Yamada et al. (2015), the authors review recent understandings from laboratory experiments on the mechanisms responsible for the heating and the acceleration of ions, as well as in the energy deposition near a magnetic null point for the electrons (for which the energy mostly comes from the electric field component perpendicular to the magnetic field).

The occurrence of solar flares predominantly in regions of sunspots has driven the analysis of the magnetic field geometry and the current systems in those regions. 
With the method proposed by Schmidt (1964), introducing a point charge to describe a magnetic field, and with the advent of the computer, kinematics of the plasma associated with different topologies could then be studied numerically and be compared with observations. In Baum \& Bratenahl (1980), the authors studied numerically in detail a system made of separatrices and null points, which allowed for comparisons with analytical works and opened the door to proper studies of reconnection phenomena in the context of solar physics.

This model, referred to as a magnetic charge topology (MCT) model in the sense that it imposes distinct unipolar regions, was refined over the years (e.g. Hénoux \& Somov 1987). It explained some flare features such as the two chromospheric ribbonshape structures appearing during certain flares (Gorbachev \& Somov 1988). Then, more complex models with several charge sources were introduced (Mandrini et al. 1991; Démoulin et al. 1993; Barnes, Longcope \& Leka 2005), so as to compare, for example, the locations of the flare kernels (i.e. brightened locations) and the topology (see Longcope 2005, and references therein).

Other methods, such as flux tubes (Sakurai \& Uchida 1977), pointwise mapping or submerged poles/dipoles models (e.g. Démoulin et al. 1994b) have also been investigated, with similar purposes to compare the complex topology features with observed features (see figure 3). Chromospheric kernels and flare ribbons were found to appear on a part of the photopheric footprints of separatrices (e.g. Hénoux et al. 1993; Mandrini et al. 1993; Démoulin et al. 1994b). They provided, over the years and with an increasing number of observations and refined techniques, convincing support for the hypothesis that magnetic energy is indeed being converted during solar flares via magnetic reconnection.

\subsection{Quasi-separatrix layers and hyperbolic flux tubes}

In Démoulin, Hénoux \& Mandrini (1994a), the authors computed the topology of several flaring regions and looked at the locations of nulls, obtained both with potential and linear force-free field models. These locations were then compared with that of energy release seen in UV and X-rays. They then found that the location of the null, or even its presence, was not necessary to explain the different locations of energy release, whereas the spatial properties of the coronal field was.

A few years earlier, Hesse \& Schindler (1988) and Schindler, Hesse \& Birn (1988) had also proposed that in a 3-D volume, magnetic field dissipation should include all effects with localised non-idealness that lead to a parallel component of the electric field along the magnetic field. Then, reconnection does not need to be associated with magnetic nulls, closed field lines or other singular structures. For magnetic reconnection to occur, there is a need to create a dissipation layer where the electric current density is important: Priest \& Démoulin (1995) and Démoulin et al. (1996a) added that such regions are created if the magnetic field connectivity is strongly distorted but can still remain continuous.

In Aulanier et al. (2005) and Pontin et al. (2005), the authors investigated the evolution of the magnetic field in a 3-D MHD simulation in the absence of magnetic null points, but with the presence of a non-ideal region formed by shearing/twisting boundary motions. They noted that the field lines would change their connections continuously in the volume with strong current concentrations. They verified, with these simulations, that in the presence of a strong $E_{\|}$component, reconnection could still happen without the presence of null points.

In the mathematical sense, null points are strictly defined as the locations where the magnetic field vanishes, and are accompanied by separatrices and separators. However, 
(a)
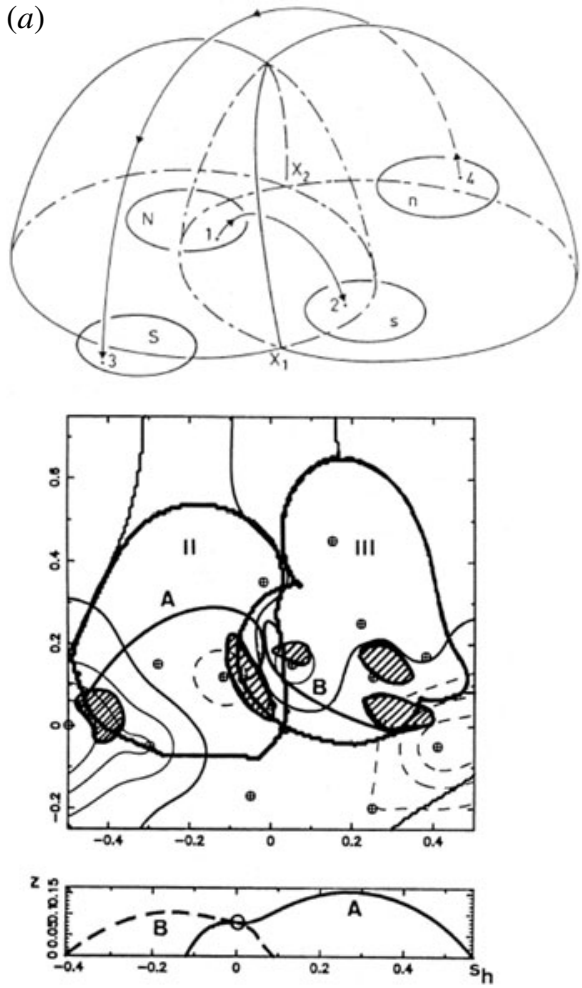

(b)
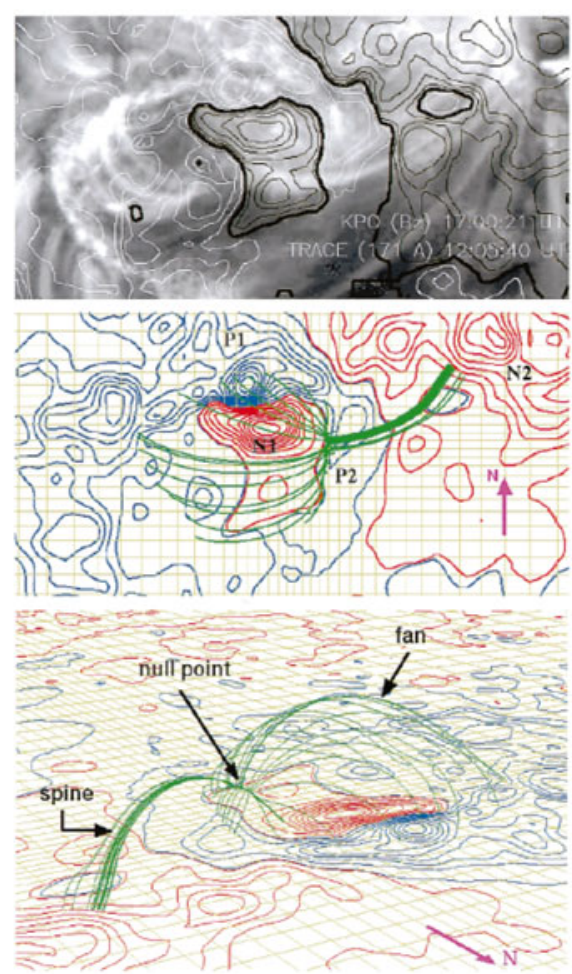

FIGURE 3. (a) Simplified schema of separatrice surfaces in the presence of two bipoles 1-2 and 3-4 (top) and comparison of their photospheric traces with the locations of $\mathrm{H} \alpha$ brightening seen during a flare, adapted from Mandrini et al. (1991). (b) Magnetic field configuration of a flaring region associated with a spine and fan structure as obtained from a magnetic field extrapolation. They can directly be compared with emissions seen in extreme ultra-violet (UV) of coronal loops, adapted from Aulanier et al. (2000).

a broader class of structures may be defined: these are quasi-separatrix layers (QSLs). When thin enough, QSLs behave physically as separatrices even though there are no true mathematical discontinuities of the field line mapping.

This generalisation to QSLs was introduced by the seminal works of Priest \& Démoulin (1995) and Démoulin, Priest \& Lonie (1996b). QSLs are defined as regions of space where the connectivity of the magnetic field is drastically changing, while remaining continuous in general. This is for example illustrated in figure 4(a) where two bipoles are shown. The pale blue and magenta crescent regions indicate the footpoints of QSLs in a schematic way. The configuration does not have a null point but as the flux concentrates in each magnetic poles, it still creates a gradient in the connectivity of the field lines.

To properly define regions of strong changes of connectivity, there is a need to introduce the mapping of field lines, which follows the connectivity of a field line from one footpoint to another in a given magnetic configuration. In the solar context, this lower boundary is introduced because of the line-tying of the field lines in the dense and slowly evolving photosphere. This boundary is in general not required to define QSLs (Démoulin et al. 1996a). Below, we keep this description because of its 
(a)

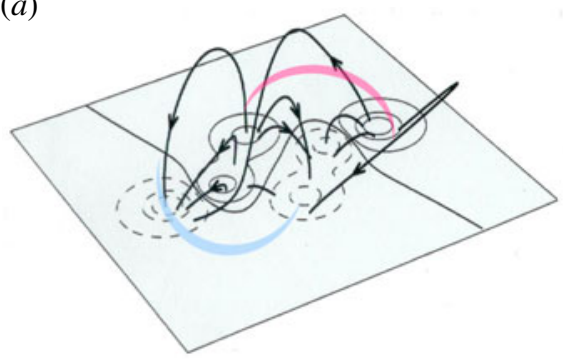

(c)

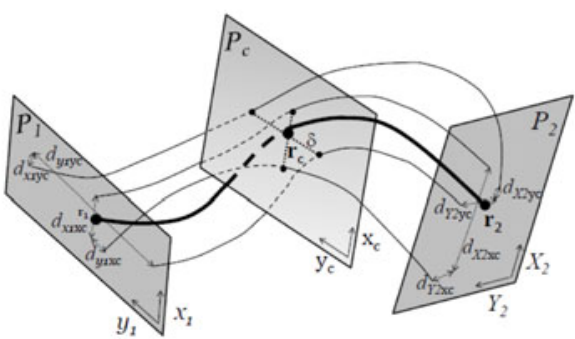

(e)

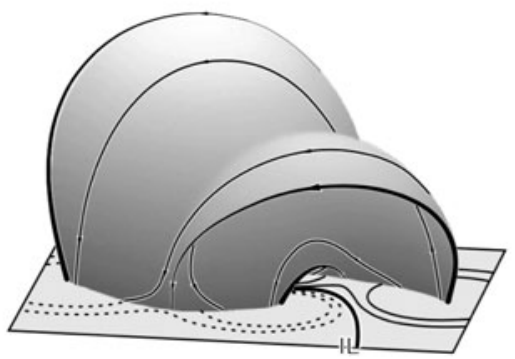

(b)

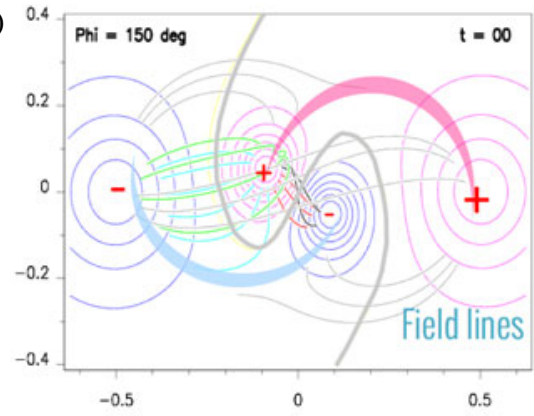

(d)

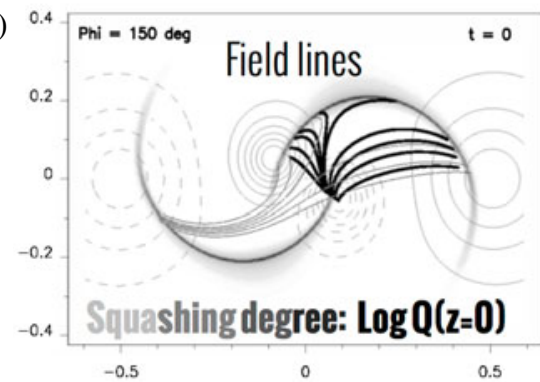

$(f)$

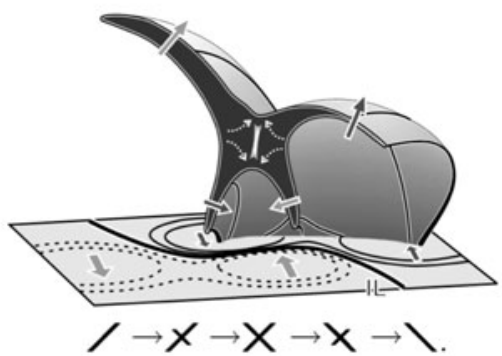

FIGURE 4. (a) A set of magnetic field lines randomly traced in a quadrupolar configuration without a null point. The trace on the lower plane of the largest gradient of connectivity, i.e. the QSLs, are shown with pale blue and magenta crescent areas. They are located within magnetic polarities (dotted and plain isocontours on the surface) of the same sign. (b) Quadrupolar configuration analysed in a numerical set-up by Aulanier, Pariat \& Démoulin (2005) where field lines are traced in different colours depending on their anchoring region. For example, the green and blue sets of field lines are departing from the same positive polarity (in magenta), but are seen to connect to the different negative polarities (blue isocontours). As such, one can trace the connectivity gradient region (in magenta). (c) The field line mapping and the squashing degree $Q$ can be calculated following the technique of Pariat \& Démoulin (2012), which is illustrated here by a generic connectivity between two local planes while the QSL trace is computed on the central plane. $(d)$ The QSLs are computed numerically: their traces on the photospheric plane are shown in gradient of grey, with the darker greys indicating higher values of the squashing degree $Q$. Two sets of field lines are added with their footpoints selected on a segment crossing the QSL trace. They show a divergence pattern characteristic of field lines across QSLs. The whole QSL volume is represented in perspective in $(e)$, for a similar quadrupolar configuration. $(f)$ A cut within the volume shows the X-shaped morphology of the QSLs, also called a hyperbolic flux tube (adapted from Titov, Hornig \& Démoulin 2002). 
direct solar application. The mapping norm was introduced (equation (4) in Démoulin et al. 1996a):

$$
N=\sqrt{\left(\frac{\partial X^{2}}{\partial x}+\frac{\partial X^{2}}{\partial y}+\frac{\partial Y^{2}}{\partial x}+\frac{\partial Y^{2}}{\partial y}\right)},
$$

where $(x, y)$ represent one footpoint location at one plane (e.g. see figure 4), and $(X, Y)$ the corresponding other footpoint of the same field line. A QSL is defined as any region where $N \gg 1$. A strongly distorted connectivity region, or QSL, means that when tracing magnetic field lines anchored in the same vicinity, their opposite footpoints will be located at very different positions in the opposite polarity (figure $4 b, d$ ).

However, the mapping norm is only defined by a neighbouring field line region in one polarity: its values therefore depend on which polarity the field lines have been chosen (it is footpoint dependent). To estimate a non-footpoint-dependent parameter, Titov et al. (2002) defined another parameter, the squashing factor $Q$. It is independent of the footpoint as it is weighed by the ratio of the vertical field component at each footpoint. Similarly to $N, Q$ provides an information on the distortion of the magnetic field connectivity. By introducing the two footpoint mappings $N_{12}$ and $N_{21}$ :

$$
\begin{gathered}
N_{12}=\sqrt{\left(\frac{\partial X_{2}^{2}}{\partial x_{1}}+{\frac{\partial X_{2}}{\partial y_{1}}}^{2}+{\frac{\partial Y_{2}}{\partial x_{1}}}^{2}+\frac{\partial Y_{2}{ }^{2}}{\partial y_{1}}\right)} \\
N_{21}=\sqrt{\left(\frac{\partial X_{1}{ }^{2}}{\partial x_{2}}+\frac{\partial X_{1}}{\partial y_{2}}+\frac{\partial Y_{1}{ }^{2}}{\partial x_{2}}+\frac{\partial Y_{1}}{\partial y_{2}}\right)},
\end{gathered}
$$

the squashing degree was defined as:

$$
\begin{aligned}
Q & =Q_{12}=\frac{N_{12}^{2}}{\left|B_{z, 1}\left(x_{1}, y_{1}\right) / B_{Z, 2}\left(X_{2}, Y_{2}\right)\right|} \\
& =Q_{21}=\frac{N_{21}^{2}}{\left|B_{z, 2}\left(x_{2}, y_{2}\right) / B_{Z, 1}\left(X_{1}, Y_{1}\right)\right|} .
\end{aligned}
$$

Then, QSLs represent the regions with the highest squashing factor $Q$. Recently, a computational method to obtain the squashing degree within a 3-D domain was proposed by Pariat \& Démoulin (2012) (see figure 4c). This was further developed in Tassev \& Savcheva (2016).

Since the QSLs form a thin volume (e.g. figure $4 e$ ), one can define the region where the field lines diverge the most in the central part of this volume. In figure $4(f)$, a transverse cut in the volume is shown, which displays 4 branches marking the characteristic shape of QSLs. This particular region was coined a hyperbolic flux tube (HFT, see Titov et al. 2002). HFTs can also be understood as the 'intersection' of two QSLs, and as such, are generalising the concept of a separator line in three dimensions in the context of QSLs (i.e. since a separator is formed by the intersection of two separatrices). Their shape is particularly reminiscent of an X-point in the transverse cut. At the bottom of figure $4(f)$ a schema indicates its shapes following the location of the cut in the volume (i.e. those shapes are obtained by doing different cuts of the HFT orthogonal to the local magnetic field). Closer to the anchoring surface, the HFT resembles a line: they are the inner regions within the pale blue and pale magenta traces shown in figure $4(b)$. Further away from the 


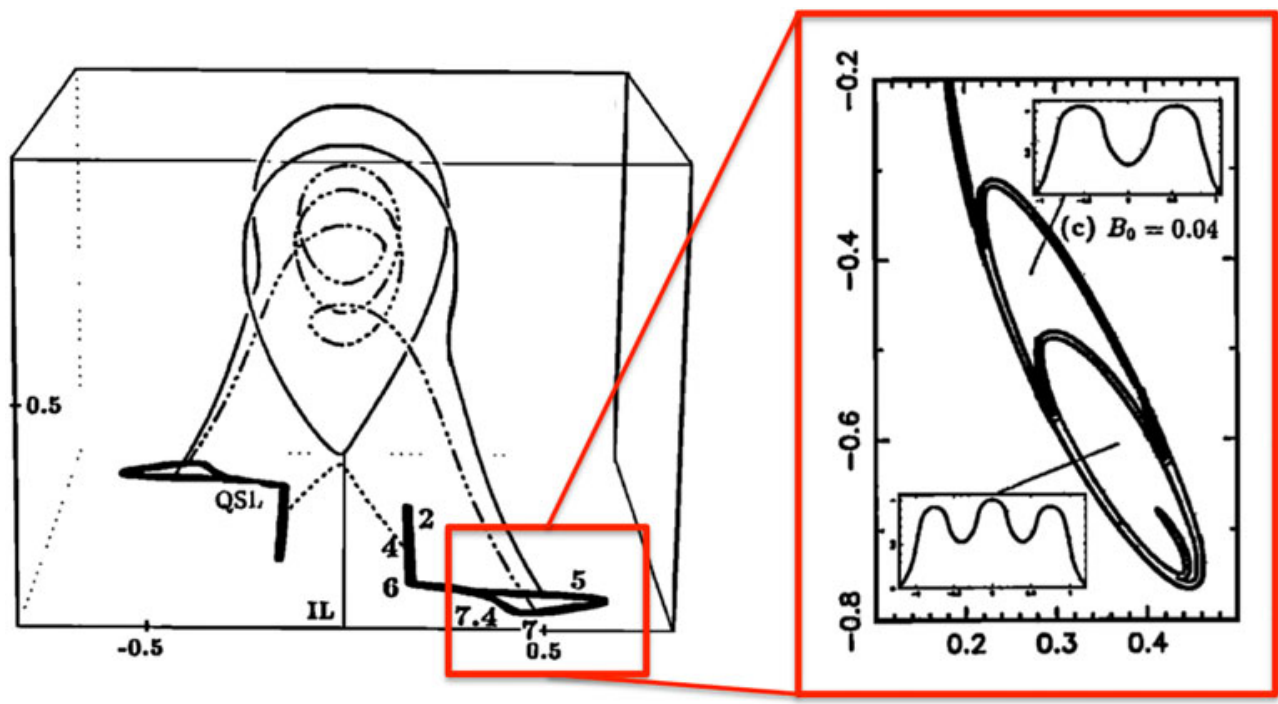

FIGURE 5. Projected view of a configuration containing a flux rope, as indicated with the dashed-dotted (three turns) and solid (one turn) twisted field lines. The small, dotted field line represent a coronal loop lying underneath the flux rope. The gradient of connectivity between these field lines is indicated with the elongated, bold lines at the photospheric level (QSL trace). Their straight part is associated with the low-lying coronal loop, while the round region is associated with the anchoring region of the twisted field lines. A zoom in the region shows a hook shape of the flux-rope anchoring region, where a higher twist corresponds to a higher number of swirls (adapted from Démoulin et al. (1996b)).

photosphere, the HFT displays an X-shaped structure. Recently, the concept of the squashing factor to define QSLs and HFTs has been extended to 'slip-squashing' factors (Titov et al. 2009) (from the discussion in Hesse, Forbes \& Birn (2005)), which allows the characterisation of the change in the magnetic field connections.

\subsection{QSLs in the presence of flux ropes: from theory to observations}

In the presence of a twisted magnetic field, the quasi-separatrix layers trace the frontier between the twisted magnetic field and the more potential surrounding field (see figure 5). Their traces on the lower boundary show a typical $J$-shape, with a straight part associated with low-lying coronal loops while the hook region of the $J$ shape is associated with the anchoring region of the flux rope. In Démoulin et al. (1996b), the authors investigated the morphology of the QSLs and especially the hook region: because the QSLs form a thin volume, that delimitates the frontier between differently connected field lines, this volume is also connected to the photospheric surface. Then, the more the magnetic field is twisted, the more complex the QSL photospheric footprint becomes: the QSL swirls around the flux rope as the twist of the structure increases (figure $5 b$ ). QSLs in the presence of flux ropes were also investigated in laboratory experiments. Gekelman, Lawrence \& Van Compernolle (2012) provided experimental evidence that QSLs indeed form in the presence of flux ropes created in a laboratory device.

Because of the strong distortion of the magnetic field line mapping at QSLs, the latter were proposed as preferential locations for current buildup (Démoulin 
(a)

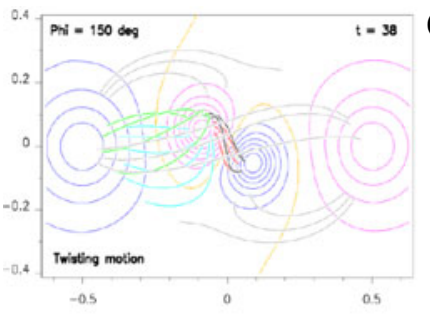

(b)

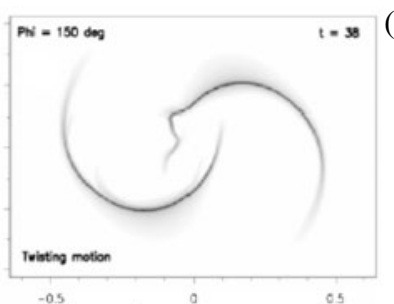

$(c)$

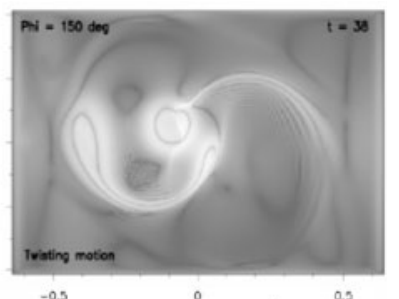

$(d)$

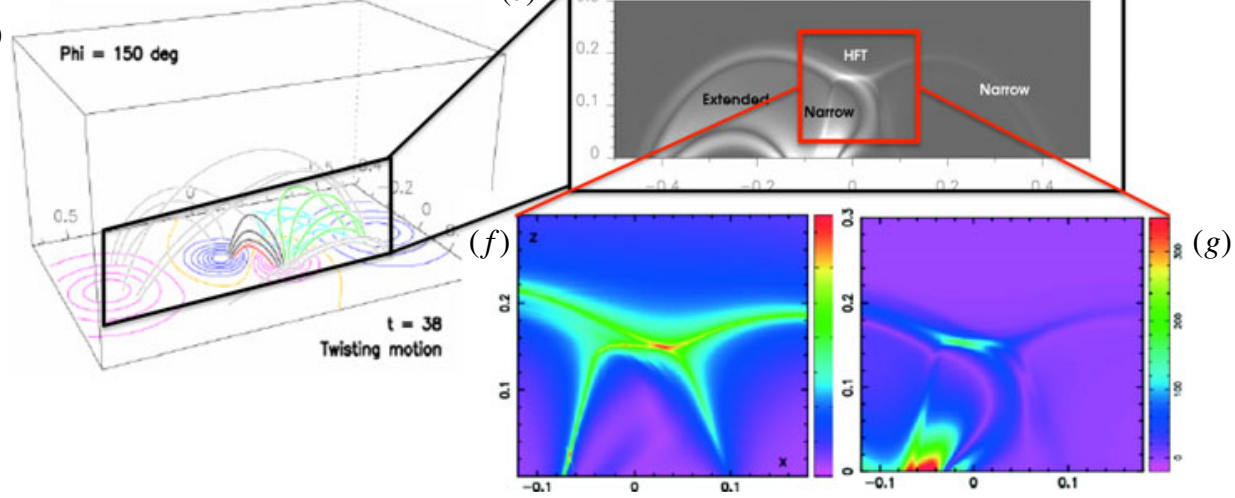

FIgURE 6. (a) Top view of a quadrupolar magnetic configuration (with two bipoles, where the positive (respectively negative) polarity is indicated in magenta (respectively blue)). A photospheric velocity field is applied as a boundary condition so as to reproduce a twisting motion in the small positive polarity. The photospheric traces of the associated QSLs are shown in $(b)$ : the highest values of the squashing degree $Q$ are shown in black. The electric currents are shown in greyscale in $(c)$, with the most intense currents shown in white. $(d)$ Side view of the configuration, with $(e)$ showing a transverse cut in the middle of the domain of the coronal current density. The strongest currents are seen to appear at the locations with the highest squashing degree or HFT, as is also shown in the colour-coded zooms of the QSLs $(f)$ and the currents $(g)$ (adapted from Aulanier et al. (2005)).

et al. 1996a; Démoulin 2005). This was investigated in numerical simulations, and several authors indeed showed the generic formation of strong current density regions at QSLs, as indicated in figure 6 (Aulanier et al. 2005; Pariat, Aulanier \& Démoulin 2006; Masson et al. 2009; Effenberger et al. 2011; Craig \& Effenberger 2014). However, an exact one-to-one correspondence between the localisation of the highest squashing degree values (HFT) and the highest current densities is not necessarily found, as was shown in Wilmot-Smith, Hornig \& Pontin (2009) and (Janvier et al. 2013). The correspondence of QSLs with regions of reconnection is not limited to MHD models generally investigated in the context of the Sun. Recent kinetic simulations in three dimensions such as by Wendel et al. (2013) have shown that QSLs are associated with areas of large gradients of parallel electric field, which provide a new understanding on the determination of reconnection sites in predominantly collisionless plasmas such as the Earth's magnetosphere. Since electric currents are extremely difficult to directly investigate in the solar corona (because of the absence of reliable direct observations of magnetic fields in the Sun's atmosphere), simulations provide a useful environment to understand the formation and the evolution of currents in the presence of QSLs, such as shown in figure 6. 
Recently, proxies of coronal currents, such as their photospheric signatures, have been used to investigate the similarities in the location, morphology and evolution of QSLs and currents. They show the correspondence expected from numerical simulations, as detailed in $\S 3.4$.

Since QSLs generalise the concept of separatrices and are associated with locations of electric currents, and hence reconnection, investigation of the topology of flaring magnetic field configurations naturally led to the search for QSLs and their relation with flares. For example, Démoulin et al. (1996a, 1997) used modelling techniques of the coronal field to reproduce magnetograms of flaring regions and computed QSL locations. In these works, the authors found that locations where $\mathrm{H} \alpha$ brightening was observed were related to locations of QSLs. Furthermore, QSLs can also be found in the presence of a null point: such a configuration was also investigated in a circular ribbon flare, using data-driven simulations (Masson et al. 2009). Since then, investigating the locations of QSLs has proved to be successful at interpreting a large variety of flaring regions (e.g. Schmieder et al. 1997; Bagalá et al. 2000; Mandrini et al. 2006; Restante, Aulanier \& Parnell 2009; Chandra et al. 2011).

From then on, the locations and the morphology of sudden flare brightenings were compared with the locations of QSLs found with a magnetic field model. Over the years, the techniques were refined, helped by higher spatial resolution instruments as well as more refined modelling techniques (such as magnetic field extrapolations). To give an example, the ribbons of eruptive flares were often compared with QSLs found in the presence of flux ropes: indeed, this type of flare often displays the presence of two flare ribbons appearing in $\mathrm{H} \alpha$ (Chandra et al. 2009), and in particular these ribbons often display a $J$-shaped structure. This morphology is very similar to the analytical shapes found for QSLs in the presence of twisted flux tubes (see above and figure 5), as well as in recent 3-D MHD numerical simulations of eruptive flares (see figure 3 in Janvier et al. (2013)).

Savcheva et al. (2012a) investigated the shape of QSLs for a magnetic field model of an active region which was associated with the presence of a sigmoid (a region where the coronal loops display $J$ - or $S$-shaped features, indicative of a flux rope, see e.g. Green et al. 2007; McKenzie \& Canfield 2008). They model the flux rope associated with the sigmoidal region using the flux-rope insertion method (see van Ballegooijen 2004). With a magnetic model of the active region, the authors could investigate the connectivity change in the volume of interest, and look in more details at the morphology of the QSLs. Some of these results are reproduced in figure 7, where it shows that the photospheric traces of the QSLs are much more complex than in analytical and simulation models, yet still displaying a hooked, $J$ shape. A transverse cut in the middle of the twisted structure also shows the presence of an $X$-shaped region that is reminiscent of the HFT (figure $7 e-h$ ). Such a work therefore provides observational evidence that HFTs and associated QSLs are generic features found in the presence of sigmoidal regions and flux ropes.

Recently, such an analysis has been extended to a variety of flaring regions and with different techniques. For example, Zhao et al. (2016) studied a flaring region and applied another technique to reconstruct the magnetic field of the region, namely, a nonlinear force-free field model (following the technique of Gilchrist \& Wheatland 2013). They found similar morphologies in the shape of the QSLs as in Savcheva et al. (2012a). As such, the morphology of QSLs in the presence of erupting flux ropes is independent of the extrapolation method. This is expected because of the structural stability of QSLs, in contrast to the presence and the location of null points and associated separatrices, (see Démoulin et al. 1994a; Janvier et al. 2016, on the 
(a)

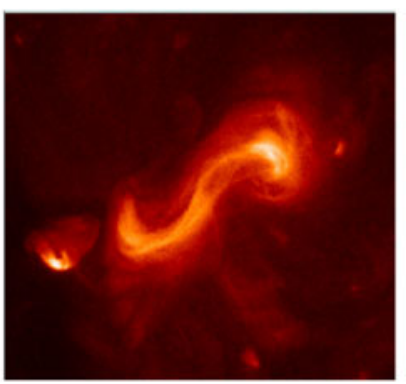

(c)

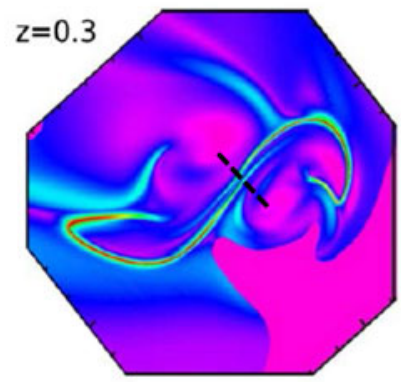

$(e)$

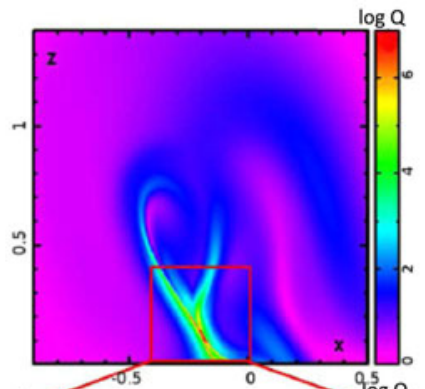

$(g)$

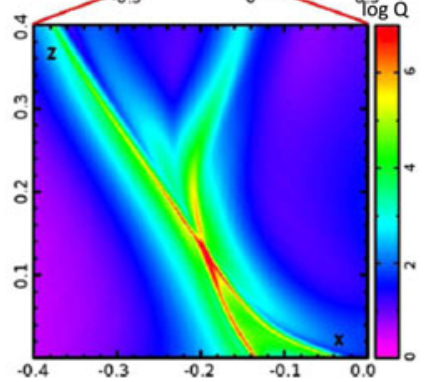

(b)

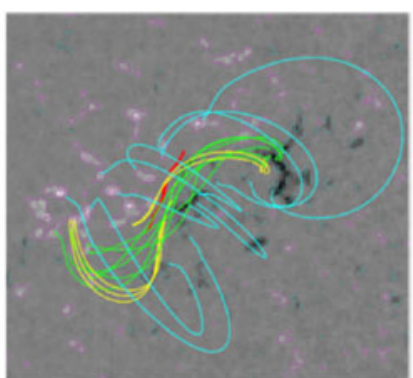

(d)

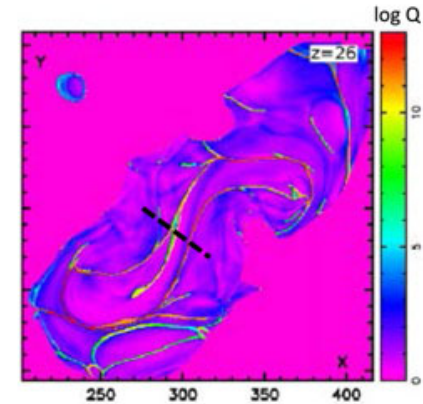

$(f)$

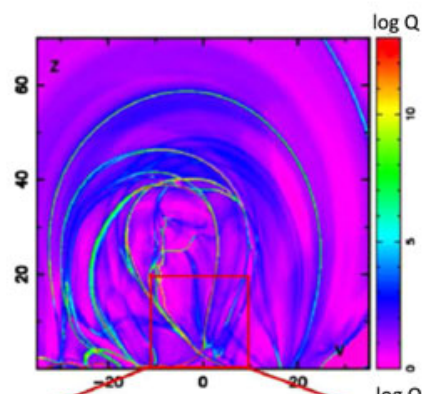

(h)

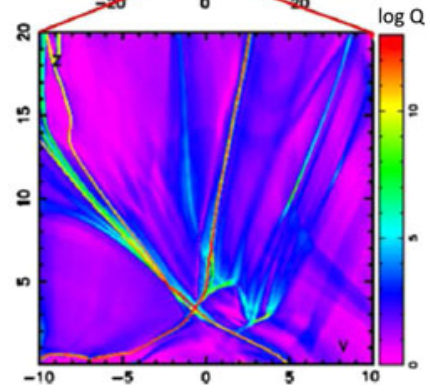

FIGURE 7. (a) Sigmoidal region seen by the XRT instrument aboard Hinode from which a magnetic model is constructed, shown with a sample of field lines in $(b)$. Panels $(c, e, g)$ show the numerical simulation of an unstable flux rope, while $(d, f, h)$ show similar plots for a magnetic configuration derived from observations $(b)$. The (near) photospheric traces of the QSLs for a numerical flux-rope simplified model are shown in $(c)$. They are compared in $(d)$ with that of the configuration created by a flux rope inserted in the extrapolated potential magnetic field of the magnetogram shown in $(b)$. They both display the typical $J$ shape expected in the presence of a flux rope. A transverse cut (dashed black lines in $c$ and $d$ ) is shown in the magnetic field numerical model $(e)$ and the extrapolated magnetic field $(f)$, where the location of the highest values of the squashing degree $Q$ is found underneath the flux rope in both cases. A zoom indicate the presence of a HFT in $(g, h)$ (adapted from Savcheva et al. (2012a)). 
influence of the extrapolations on the stability of null points and QSLs). Furthermore, the shapes of the QSLs can be directly compared with that of the flare ribbons.

Then, evolving the magnetic model of an active region, for example by an MHD evolution or a magnetofrictional relaxation (in the case of an unstable flux rope), also allows the investigation of the evolution of QSLs. The multiple time sequences of these evolutions can provide snapshots that can be compared with observations of flare ribbons, as presented in Savcheva et al. (2015, 2016), Janvier et al. (2016).

As such, the search for QSLs in a magnetic field reveals the coronal locations of the dissipation, as well as a tool to interpret the photospheric signatures of reconnection as seen with flare ribbons.

\section{Dissipation layer}

\subsection{Dissipation process}

The first developments in the theory of magnetic field reconnection were accompanied by a description of the dissipation process taking place in current layers. The diffusion terms appear in Ohm's law as non-ideal terms. In its simplest description (resistive MHD for a single fluid), the non-ideal departure is described with $\eta \mathbf{J}$ :

$$
\boldsymbol{E}+\boldsymbol{v} \times \boldsymbol{B}=\eta \boldsymbol{J},
$$

where $\eta$ is the plasma resistivity (a measure of the collisionality of the plasma, see Spitzer (1956)) and $\boldsymbol{J}$ is the electric current density vector. The effect of finite conductivity on the diffusion of magnetic fields was already investigated for several configurations by Parker \& Krook (1956). However, simply considering the diffusion of magnetic fields in the corona provides time scales (years) that are considerably larger than that of flares (minutes). Then, Petschek (1964) introduced another reconnection model where he instead considered a much shorter current sheet, which provided the much greater inflow speed needed for fast reconnection. In his model, he overcame the elongation of the current layers (from an $X$ shape to a double Y shape) by invoking the appearance of oblique, magnetohydrodynamic, standing shock waves which develop at the edges of the current layer. Then, the reconnection rate is enhanced by hydromagnetic actions (since the waves allow magnetic energy conversion) outside the diffusion region, while the ohmic dissipation only occurs in the very small current layer. However, its mechanism was doubted (Green \& Sweet 1967) and later proved to not be reproducible in numerical simulations either (Biskamp 1986), unless when some variation in the resistivity was accounted for (see Baty, Forbes \& Priest 2009). Further developments of the Sweet-Parker and Petschek models were compared, such as by Kulsrud (2001), who investigated analytically the similarities between the Sweet-Parker and the Petschek models. Both are found to have a similar reconnection rate in case of constant resistivity due to the imposed condition that the length of Petschek's diffusive layer is not a free parameter, a condition that was not taken into account in the original work. The Sweet-Parker current sheet, which describes a stationary model, is quite restrictive compared with the possible evolutions of current sheets, as described below in $\S 3.2$. We also refer the reader to the historical evolution and recent advances deriving from the Sweet-Parker configuration discussed in Loureiro \& Uzdensky (2016).

A more complete description of the physical dissipation mechanism in current layers can include ambipolar diffusion, which is effective in partially ionised gases of sufficient density (Piddington 1954b). This process is related to the drift of the plasma 
with respect to the neutrals in the presence of a magnetic field (see Zweibel 2015, for more insights on the phenomenon and its importance in astrophysical systems). The ambipolar diffusion does not itself lead to magnetic reconnection, but as it squeezes the magnetic field lines together, they end up 'piling up', permitting a more efficient dissipation (see also Parker 1963). Ambipolar diffusion has recently been revisited in the context of reconnection in the chromosphere, where the plasma is dense (see e.g. Leake, Lukin \& Linton 2013). However, it is dubious that ambipolar diffusion may have a strong role in the corona since the density there is rather low and the corona is fully ionised.

The effects of turbulence on reducing the plasma conductivity were already pointed out by Sweet (1950). Wentzel (1963) proposed also early on that the increased dissipation due to turbulence can increase the magnetic annihilation rate. Plasma experiments such as those conducted by Baum \& Bratenahl (1976) then showed direct observations of rapid magnetic field reconnection, and transition from slow to fast reconnection due to an anomalous resistivity, validating such reconnection models. The effect of anomalous resistivity was studied numerically in different current sheet configurations: it was for example shown to account for the transition from a Sweet-Parker-like current layer to a Petscheck-type current layer (Baty et al. 2009; Baty, Forbes \& Priest 2014). The effects of stochastic magnetic fields were also shown to increase the reconnection rate (e.g. Lazarian \& Vishniac 1999).

A large body of work has investigated the reconnection mechanism at small scales. Effects such as the Hall term (Piddington 1954a), the ion inertia term and the electron pressure density can play a role in the reconnection mechanism and can change the energy partition. The relevant Ohm's law in such a case is as follows:

$$
\boldsymbol{E}+\boldsymbol{v} \times \boldsymbol{B}=\eta \boldsymbol{J}+\frac{1}{e n} \boldsymbol{J} \times \boldsymbol{B}-\frac{1}{e n} \nabla \cdot \boldsymbol{P}_{e}-\frac{m_{e}}{e} \frac{\mathrm{d} \boldsymbol{v} e}{\mathrm{~d} t},
$$

where the first term (on the right-hand side of the equation) is the plasma resistivity term, the second the Hall term, the third the electron pressure tensor and the remaining term represents the electron inertia.

These last three terms arise where the traditional MHD formalism breaks down for a current sheet that is thin enough, i.e. the diffusion region becomes thinner than the ion skin depth (in such a case, we generally refer to reconnection as being collisionless). Note that adding more terms to Ohm's law means that the physics related to the ions and the electrons is more complete than a description where the plasma is considered as a single species fluid, as can be seen by comparing (3.1) and (3.2). For example, the Hall term arises when magnetic drifts are considered: this relates to the motion separation between electrons and ions, i.e. electric currents, which then need to be added as a correction to Ohm's law (the $(n e)^{-1} \boldsymbol{J} \times \boldsymbol{B}$ term, see numerical developments in Huba 2003). We note that a careful interpretation of the Hall term is needed, since the term itself is not a magnetic field connectivity breaking term. Indeed, the Hall term decouples the ions and the electrons, and the magnetic field remains tied to the electrons (meaning that at the electron scale, the magnetic field still remains 'frozen-in'). The importance of small-scale effects was pointed out by Birn et al. (2001), where the authors investigated the effects of different numerical set-ups with different expressions of the Ohm's law. In particular, any implementation (MHD or particle-in-cell (PIC)) containing the Hall term gave similar results, contrary to the simple resistive MHD description (see figure 8a). The decoupling of the ions and the electrons are shown in figure $8(b)$, where the diffusion region is defined 
(a)

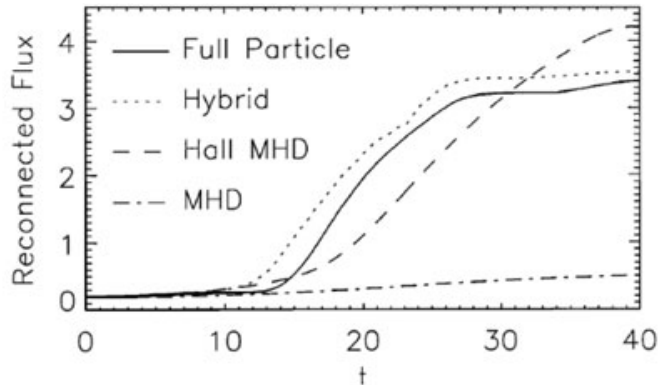

(b)

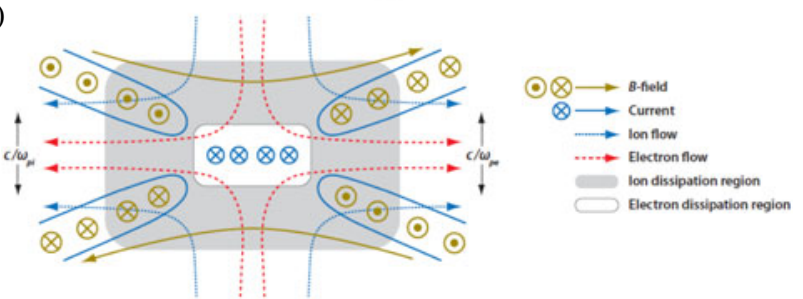

FIgURE 8. Comparison of resistive MHD and kinetic descriptions of the current layer. (a) Results of the Geospace Environmental Modeling (GEM) magnetic reconnection challenge, where several codes (MHD and PIC) were tested to investigate the effects of the nonlinear terms described in the generalised Ohm's law. It was found that codes that include the Hall term did not differ much one from another, while a conventional resistive MHD description of reconnection did not agree with all the other results. Here, the time evolution of the reconnected flux in those simulations are shown to indicate the differences (adapted from Birn et al. (2001)). (b) Description of the magnetic field geometry in collisionless reconnection, where the flows of the ions and the electrons are decoupled in the diffusion area (adapted from Zweibel \& Yamada (2009)).

differently (with different physical effects) for the ions and the electrons. Studies of small-scale effects therefore include Hall fields (Drake, Shay \& Swisdak 2008), anisotropic pressure (Birn \& Hesse 2001), electron dynamics and viscosity (Ma \& Bhattacharjee 1996; Hesse, Kuznetsova \& Birn 2004; Cai \& Li 2008), while reviews of these effects can also be found in Porcelli et al. (2002), Shay et al. (2004), Büchner (2007).

\subsection{Current layer formation and evolution}

In the Sweet and Parker mechanism (Sweet 1956; Parker 1957b; Sweet 1958a), and as well as in the Petschek mechanism (Petschek 1964), reconnection is considered analytically in a steady-state current layer, treated as a boundary layer where the plasma outside of this region is considered as current free and perfectly conducting. Such models are useful in obtaining the magnetic energy conversion (or reconnection) rates (Parker 1963), as well as providing the geometrical characteristics of the current layer (Priest \& Raadu 1975; Tur \& Priest 1976). However, in the works of Green \& Sweet (1967) and Petschek \& Thorne (1967), the authors had to modify the original Petschek reconnection scenario (e.g. with additional waves) as they recognised that the region where ohmic diffusion takes place at the intersection of two shocks is too thin to be hydrodynamic. Friedman \& Hamberger (1968) also pointed out that the current density in Petschek's original model violates the two-stream instability criterion. 
Finally, Syrovatskii's work (Syrovatskii 1966) on dynamic dissipation in current layers throws some doubt on whether a quasi-steady state can be achieved at all.

It is necessary to remind the reader that reconnection in such steady-state configurations is driven by boundary motions: the magnetic field is forced to move into the dissipation layer by a driving inflow around the reconnecting region (Hahm \& Kulsrud 1985; Wang, Ma \& Bhattacharjee 1996). The rate of reconnection or energy conversion is therefore dictated by the inflow of magnetic flux brought to the dissipation region. The dynamics of the coronal field can infer flows which velocity fields drive/enhance the reconnection in the current layer. Therefore, a Sweet-Parker regime is often a misnomer when describing reconnection processes occurring in the Sun's corona, since the inflows from the surroundings of the reconnection region are not themselves steady state. How the reconnection rate is actually determined by boundary conditions is still poorly understood (see the discussions in Comisso \& Bhattacharjee 2016). Recently, Forbes et al. (2013), Baty et al. (2014) have argued that the rate of reconnection is more likely to be controlled by the non-uniformity of the normal magnetic field in reconnecting layers rather than by the boundary conditions.

Steady-state, driven reconnection models lose the description needed to explain the onset of flares. Indeed, as was already recognised by Gold \& Hoyle (1960), it is important for a model to include the suddenness of flaring events. In particular, they acknowledged the necessity of force-free fields to progressively store free magnetic energy, which exist prior to the flare. They also recognised the need to have a storage mechanism and release mechanism, invoking the existence of instabilities.

The instability of current layers gained some attention in the early stages of plasma laboratory experiments, especially because they were found to be detrimental to the realisation of fusion, the process to harness a star-like energy. Then, Furth, Killeen \& Rosenbluth (1963) proposed that a resistive instability takes place in the current layer, which leads to a readjustment of the magnetic field in a configuration of lower energy. This lower state is characterised by the presence of magnetic islands (2-D transverse cuts of 3-D twisted flux ropes, appearing as nested magnetic field lines). As it gives a shredded structure to the current layer, this instability was coined the tearing instability. Furthermore, the interest in this mechanism for solar flare application lies in the fact that the time scale of the tearing instability was much smaller than a simple resistive diffusion of the equilibrium (although the instability grows more slowly than MHD instabilities, as $t_{A}<t_{T}<t_{R}$ where $t_{A}$ is the MHD time scale (Alfvén time), $t_{T}$ the tearing instability time scale and $t_{R}$ the resistive diffusion time scaling with $\eta^{-1}$ ). Putting numbers for the solar corona, Jaggi (1963), Sturrock (1968), Kliem (1995) found that the e-folding time of the instability was small enough to account for the time scale generally associated with solar flares (although this is only valid in the case of extremely small scales, see discussions in Del Zanna et al. 2016), while Spicer (1981) put forward this instability as a possible mechanism for particle acceleration. These findings were nonetheless challenged by the incapacity of a single tearing layer to provide the necessary current dissipation to account for the thermal emissions seen in flares (e.g. La Rosa 1990). A more complex system of small-scale current layers (or multiple tearing layers) was proposed instead. Simulations in 2.5 dimensions (i.e. with an invariant direction) of current sheets in the context of solar flares were also recently investigated (Shibata \& Tanuma 2001; Lynch et al. 2016), where the breaking up of the current layer in multiple plasmoids was shown. The connection between plasmoids investigated in numerical simulations, whether two- or three-dimensional, and observations of solar flares, are discussed in more details in $\S 4.3$. 
A variety of resistive instabilities have since then been studied, such as the resistive kink (with mode number $m=1$, also coined fast tearing as its growth rate scales with $\eta^{1 / 3}$, see e.g. Rosenbluth, Dagazian \& Rutherford 1973; Hazeltine, Meiss \& Morrison 1986; Waelbroeck 1989; Watanabe, Sato \& Nakayama 1995). Although the resistive kink instability has been extensively studied in the context of tokamak plasmas, kink modes (resistive in nature) can be put forward to explain the onset and subsequent magnetic energy dissipation of eruptive flares. This was discussed in Hood \& Priest (1979) for line-tied field lines (relevant in the context of solar flares), and numerical simulations of kink unstable flux ropes were compared with observations in Török \& Kliem (2005). Furthermore, multiple tearing instabilities can greatly enhance the reconnection rate in the nonlinear regime due to coupling between islands (where the reconnection outflow of a magnetic island can increase the inflow of a nearby reconnection site, e.g. Pritchett, Lee \& Drake 1980; Ishii, Azumi \& Kishimoto 2002; Bierwage et al. 2005; Wang et al. 2007; Janvier, Kishimoto \& Li 2011; Wang, Yokoyama \& Isobe 2015). Finally the effect of line-tying, relevant in the context of coronal magnetic field, was investigated in Delzanno \& Finn (2008), which found that the growth rate scaled with $\eta$ for small structure lengths (compared with the system size), while it was tearing-like for long structures. This may be of interest considering different reconnecting coronal loops in the context of say, nanoflares involving small loops versus the larger structures generally seen in flares. It should also be noted that recently, the tearing mode has been revisited in the context of current sheets with large aspect ratios. In such cases, it is possible to find current layers for which the tearing mode growth rate is of order unity and independent of the Reynolds number: such modes are coined 'ideal tearing modes' and are interesting to consider in the context of solar flares (Pucci \& Velli 2014; Landi et al. 2015; Del Sarto et al. 2016; Del Zanna et al. 2016).

\subsection{Current layers associated with flux ropes}

Global evolutions of current layers, in the context of solar flares, can be investigated with 3-D simulations. In the following, we focus on current layers during eruptive flares. In this context, the presence of QSLs is associated with the presence of an eruptive flux rope, as was discussed in $\$ 2.2$. For example, in a series of papers, Aulanier et al. (2010, 2012) and Janvier et al. (2013) investigated the evolution of a torus-unstable flux rope and the underlying mechanisms of 3-D reconnection. It was shown that the upward motion of the unstable flux rope away from the solar surface stretches the surrounding overlying arcades. This expansion motion is shown in figure $9(a-c)$, where field lines are drawn at three different times during the evolution of the flux-rope eruption (these lines have one fixed footpoint chosen at the beginning of the simulation).

As it is quite hard to discern the different structures in the volume, the authors performed an analysis on both the QSLs and the current density structures within the simulated volume, which are shown respectively in figure $9(a-f)$. Both the QSLs and the high current density regions are co-spatial. Throughout the flux-rope evolution, the QSLs draw the frontiers between different connectivity region: the teardrop-shaped structure (best seen in $a, b, d, e, g, h$ ) indicates the volume associated with the flux rope (see also in $\S 2.2$ ), while the $\Lambda$ region underneath marks the area where the flare loops are formed. Field lines filling the rest of the volume are overlying and surrounding arcades.

The current layer is present along parts of the QSLs. This confirms previous analyses where current layers form where QSLs exist, but also that QSLs can exist 
(a)

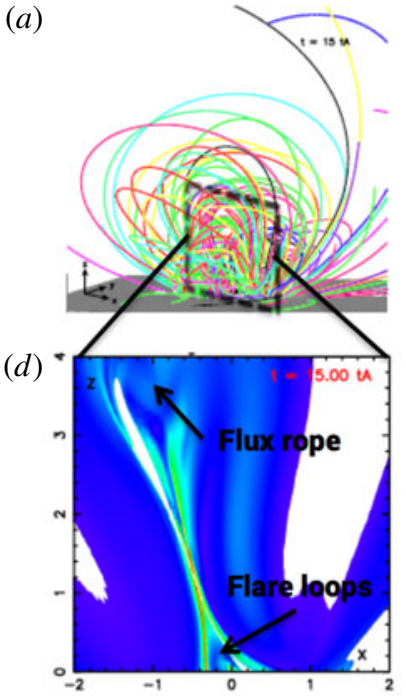

$(g)$

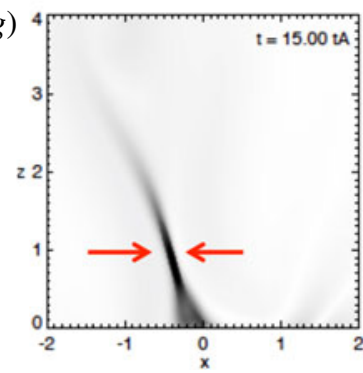

(b)

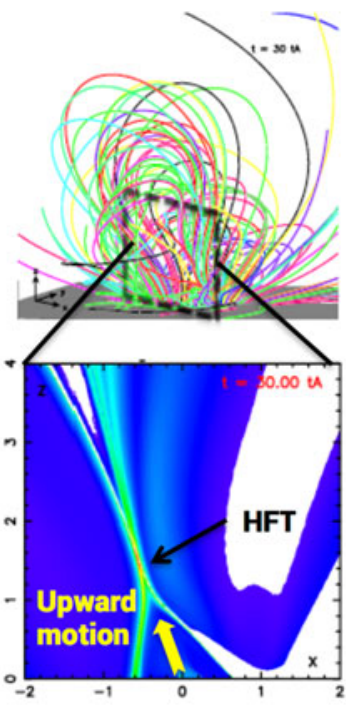

(h)

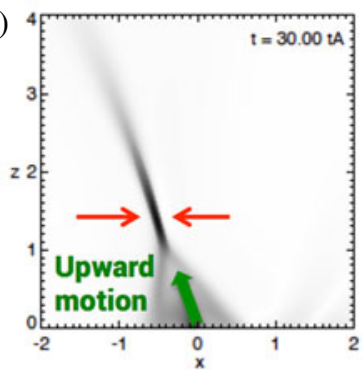

(c)

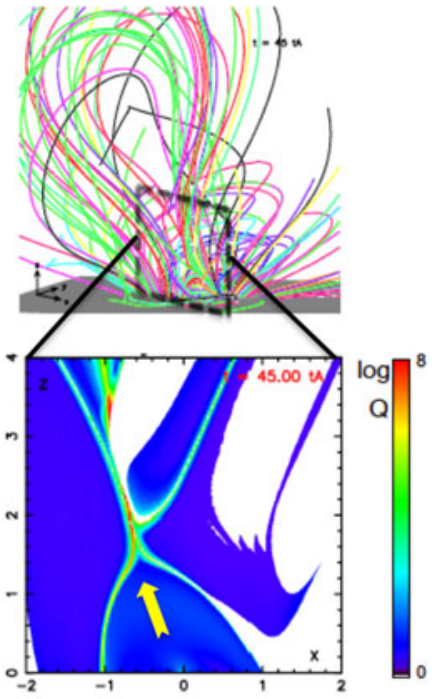

(i)

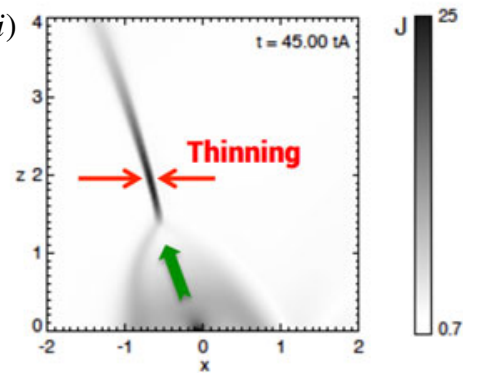

FIGURE 9. Three-dimensional representation and vertical cuts of an erupting flux rope. Results of the numerical simulation of a torus-unstable flux-rope expansion with the OHM code (Aulanier et al. 2012). ( $a-c)$ Field lines showing the expanding magnetic field as time passes by (the times represented here are $t=15 t_{A}, 30 t_{A}, 45 t_{A}$ ). A 2-D transverse cut (black dashed lines in $a-c$ ) of the QSLs is shown, for all three times, in $(d-f)$. The QSLs delimitate different magnetic field domains related to the flux rope, flare loops and surrounding field. The region of the QSLs where the magnetic connectivity changes the most is indicated as the HFT (see $§ 2.2$ ). A similar cut for the volumic current density $J$ is shown in $(g-i)$. The time evolution shows a thinning of the central current layer (indicated with red arrows), with an increased current density. The reconnection region and the top of the reconnected field lines move upward as time passes, as indicated with the yellow and green arrows (adapted from Janvier et al. 2013).

without being necessarily related to the current layers (e.g. the teardrop-shaped region is not seen in the current density transverse cut in figure 9). Interestingly, QSLs and current layers have a comparable evolution. First, their shapes are seen to evolve similarly: as time passes by, the inversed teardrop-shaped region moves upward, stretching the QSLs and the current region upward, while the $\Lambda$-shaped flare loop region grows (both are represented by the yellow and green thick arrows in figure 9). Furthermore, the current layer formed underneath the teardrop structure thins (indicated with red arrows in figure $9 g-i$ ) while the current density and squashing degree $Q$ both grow in values. The location of the highest current density is nearly 
(a)

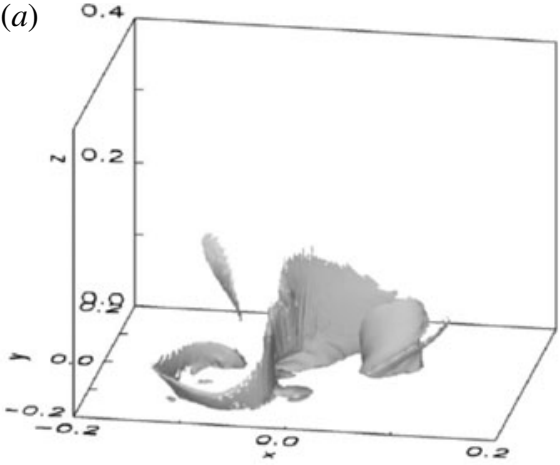

(b)

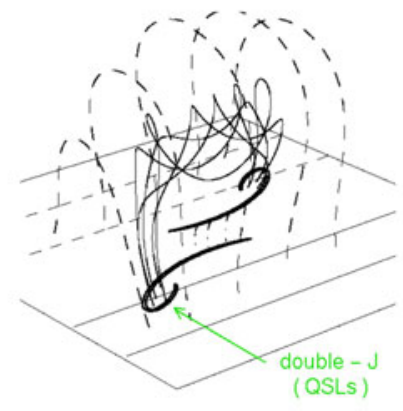

(c)

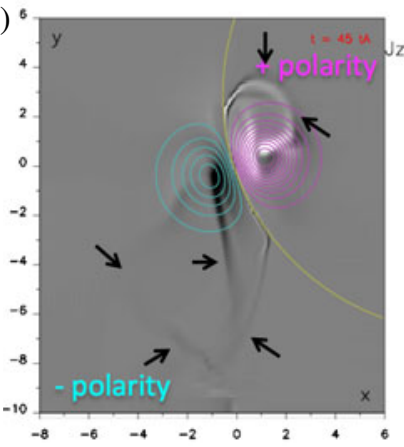

$(d)$

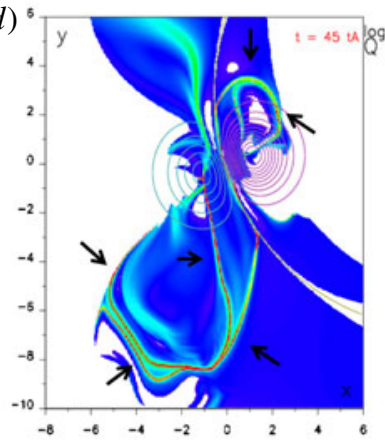

$(e)$

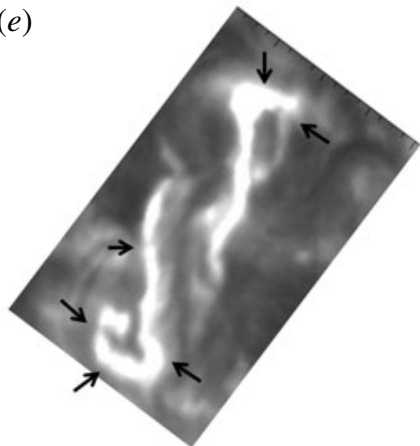

FIGURE 10. Traces on the photospheric boundary of the QSLs and current density for an erupting flux rope. (a) The 3-D volume of the current layer during a flux-rope ejection, as simulated by Kliem et al. (2013) and similar to the simulation of Aulanier et al. (2012) and Janvier et al. (2013). (b) A model of a flux rope (solid think line) underneath overlying arcades (dashed lines), showing the hooked, J-shaped QSLs (thick lines), as was first investigated in Démoulin et al. (1996b). (c) Top view of the photospheric $(z=0)$ footprints of the vertical component of the current density vector $J_{z}$ in greyscale for the flux-rope eruption simulation of Aulanier et al. (2012). The magnetic polarities are shown in magenta (positive) and cyan (negative). (d) Same view for the photospheric footprints of the QSLs. The similar $J$ shapes for the current density and the QSLs are shown with the black arrows. (e) J-shaped flare ribbons during an eruptive flare (Chandra et al. 2009).

co-spatial with that of the highest values of $Q$ which define the so-called HFT (although not exactly identical, as shown in figure 2 of Janvier et al. (2013)).

It is important to understand that in three dimensions, the current layer has a finite volume. The extent of this volume was shown in Kliem et al. (2013), who performed an MHD evolution of an extrapolated unstable coronal flux rope. It is reproduced in figure 10(a), where the volume shows a similar structure as that found with the OHM simulation of Aulanier et al. (2012), Janvier et al. (2013). Figure 10(a) shows that the volume maps all the way down to the bottom boundary or photosphere, and reaches its highest height in the centre of the volume (similar to the transverse cuts of figure $9 g-i)$. The $3-\mathrm{D}$ volume is rather thin, and displays an $S$ shape. This shape is reminiscent of the double $J$ found for the QSLs in the presence of flux ropes (figure $10 b$ and $\$ 2.2$ ). In figure $10(c, d)$, the photospheric $z$-component of the current density, $J_{z}$, is shown, along with the footprints of the QSLs. These are top views of the same structures shown in figure $9(d-i)$. Here, both the current density and the 
QSLs show the same structure, with the expected $J$ shapes, which are reminiscent of the flare ribbons typically seen during eruptive flares (figure 10e).

In such simulations, the current layer is not well resolved: this can be seen in figure 9, where the HFT region is much narrower than the current layer thickness. Indeed, the resistivity is quite large compared with solar values (to ensure numerical stability), so that the current diffuses much more than what would be expected in reality. Therefore, it remains difficult to properly address the physics of the current layer evolution, with respect to the development of instabilities such as the tearing mode. Recent investigations by Nishida, Nishizuka \& Shibata (2013) and Wyper et al. (2016) show studies of the behaviour of the 3-D current layer, with multiple shredding and plasmoid formation as would be expected from the 2-D well-studied evolution. However, the complexity of the configuration in three dimensions (see figure 4 in Nishida et al. (2013)) shows that it is difficult to properly define the equivalent of magnetic islands seen in two dimensions. This is because in three dimensions, plasmoids are not bounded by flux surfaces in the 2-D system (see e.g. Daughton et al. 2011). Still, this remains promising for further studies to understand the behaviour of the current volume and link it to observational consequences of reconnection (see $\S 4.3$ ).

\subsection{Electric currents in observations, and their associations with flare ribbons and QSLs}

Already in the 1960s, changes in the magnetic field during flares were already reported by Severny (1964a). However, the temporal resolution at this time meant that each measurement was done far from the flare time, so that it remained difficult to conclude whether those changes were really flare related or intrinsic to the evolution of the active region. Over the years, refinement in the spatial and temporal resolutions have brought more and more evidence of changes in the photospheric magnetic field during a flare. Some authors reported a decrease in the longitudinal magnetic field (e.g. Kosovichev \& Zharkova 1999), while some others (see Sudol \& Harvey 2005; Petrie \& Sudol 2010, and references therein) reported both increase and decrease depending on the flaring regions investigated. Local changes in the horizontal photospheric magnetic fields were also recently reported (e.g. Wang et al. 2002; Sun et al. 2012; Wang et al. 2012; Petrie 2013), while in those studies, the vertical field component does not change much.

Studies of electric currents during flares were already made with the first magnetograms. In particular, Moreton \& Severny (1968) already showed then that flare knots (defining the small areas of intense $\mathrm{H} \alpha$ emission) arise near regions where strong electric current density were reported. Other authors have also reported extended patches of strong current densities with ground-based magnetograms (e.g. Hagyard 1988; Hofmann, Grigor'ev \& Selivanov 1988; de La Beaujardiere, Canfield \& Leka 1993). Recently, Janvier et al. (2014a) and Musset, Vilmer \& Bommier (2015) investigated the evolution of strong electric current density regions with a higher spatial and temporal resolution given by the Helioseismic and Magnetic Imager (HMI) aboard the Solar Dynamics Observatory (SDO) mission (see the details of the instrument in Schou et al. (2012)).

With the 12 min cadence measures of the X-class flare seen on 15 February 2011 in AR11158 (SOL2011-02-15T01:53), Janvier et al. (2014a) used an inversion method of the observed polarised spectral profiles to obtain the three components of the magnetic field (e.g. Bommier et al. 2007). HMI data show that the photospheric 

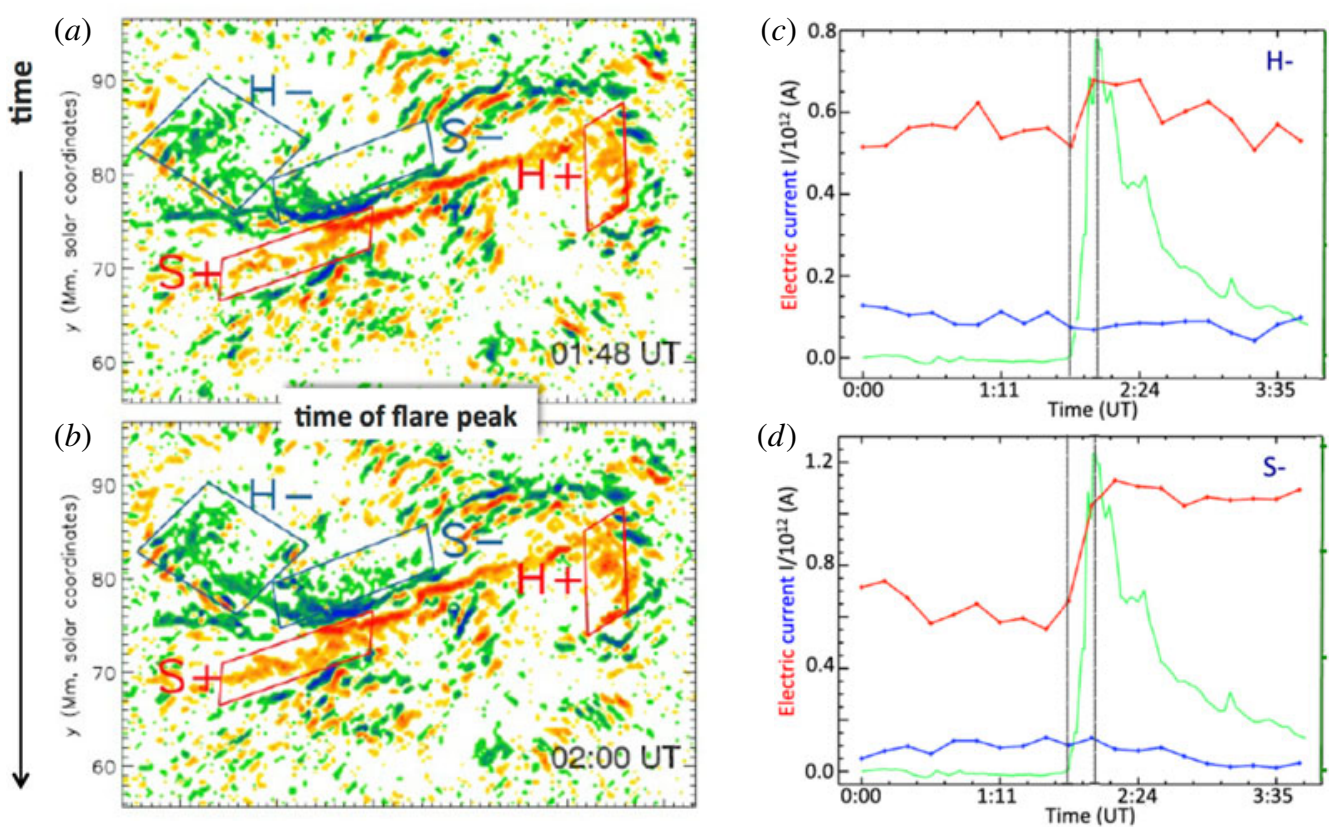

FIGURE 11. Photospheric map (where the background noise has been removed) of the vertical $(z)$ component of the current density at 01:48 UT $(a)$ and 02:00 UT $(b)$ on 15 February 2011 when an X-class flare was recorded. The time of the flare peak, from GOES Soft X-ray bands, is between the two snapshots shown here. The four squares are marked as areas where the strongest changes are seen before and after the flare impulsive phase. The regions marked with $S$ indicate the straight part of the $J$-shaped current ribbon, while those marked with $H$ indicate the hook region of the $J .(c, d)$ The light curve in the $335 \AA$ filter of the Atmospheric Imaging Assembly (AIA) instrument aboard the Solar Dynamics Observatory (Lemen et al. 2012) is shown in green, while the time evolution of the electric current $I$ (computed over the regions $\mathrm{H}-$ and $\mathrm{S}-$ defined in panels $a$ and $b$ ) is shown in red (respectively blue) for the direct current (respectively return current, see text for details). The figure is adapted from Janvier et al. (2014a).

traces of the vertical current component $J_{z}$ are similar to what is predicted from the shape of QSLs in the presence of flux ropes (see figure 10). This shape is shown in figure 11 $(a, b)$, where the $J_{z}$ component displays the expected thin and elongated $J$ shape. Interestingly, the current distributions are different, as they represent a time before $(a)$ and after $(b)$ the onset of the flare. In particular, in the regions highlighted with parallelograms (blue and red, marked $\mathrm{H}-, \mathrm{S}-, \mathrm{H}+, \mathrm{S}+$ ), one can see an increase in the current density values: the current 'ribbons' are seen to elongate and spread, similar to the outward motion away from the inversion line of observed extreme-ultraviolet (EUV) ribbons. It is also possible to integrate the current density in the different highlighted areas in order to obtain the time evolution of the electric current $I=\iint I \mathrm{~d} x \mathrm{~d} y$. Its evolution is shown in the two areas $\mathrm{H}-$ and $\mathrm{S}-$, where one can see that the sudden increase in the direct current is associated with that of the light curve, i.e. with the impulsive phase (shown in red in figure $11 c, d$ ). A direct current is defined as parallel/antiparallel to $\boldsymbol{B}$ for a positive/negative current helicity. The increase in the current density remains rather stable during the decreasing phase of the flare. 
This result may at first sound counter-intuitive, since we would expect that the current density decreases, as a flare leads to a reconfiguration of the magnetic field where the free energy has decreased, i.e. closer to a potential state. However, there is a competition between two mechanisms: the ideal instability which triggers the field evolution, and the subsequent reconnection. The first mechanism implies the increase of the current magnitude in the current layer, a generalisation of what is known in two dimensions (Lin \& Forbes 2000). Then, an increase of current magnitude is expected when reconnection cannot dissipate fast enough the accumulated current density in the current layer. With recent observations, as shown in figure 11, we are now able to witness the collapse of the current layer and the implied increase in electric current, which corresponds to the onset of the flaring phase (as fast reconnection is then triggered).

Following up on this study, Janvier et al. (2016) investigated the more complex flaring region of 6 September 2011 (SOL2011-09-06T22:20). This region, as shown in figure 12, shows a complex network of flare ribbons compared with a typical two-ribbon eruption. A careful investigation with a magnetic field reconstruction model showed that a flux rope was present, embedded in a topology reminiscent of a null point configuration, although a stable null point was not found in the extrapolations. A parasitic polarity is responsible for the spine/fan-like configuration, similar to what is normally found in the presence of magnetic null points (see Masson et al. 2009, for an example of flare ribbons in the presence of a magnetic null point). The connectivity mapping of the domain was provided by the technique developed in Pariat \& Démoulin (2012) and a novel technique for QSL calculations in three dimensions as described in Tassev \& Savcheva (2016). As such, when the flare started, the flux-rope ejection was accompanied with reconnection occurring at the quasi-spine/fan configuration, with some flare ribbons tracing the photospheric traces of the fan dome. Still, the two opposite ribbons associated with the erupting flux rope displayed the typical $J$ shape, showing that even in complex configurations, flux-rope eruptions display similar outcomes as expected from analytical/numerical models of twisted configurations.

Then, the QSLs computed from the magnetic field were compared with the locations of the flare ribbons seen in different filters, as well as the locations of the electric current density regions discussed above. It was then found that the QSLs associated with the flux-rope location (as found with the flux-rope insertion method, described in van Ballegooijen 2004) displayed a similar $J$-shape feature as those reported in analytical and numerical studies.

Following this analysis, the magnetic model of the flaring active region was forced to evolve via a magnetofrictional code. This technique has been applied to a series of active regions, as reported in Savcheva et al. (2016). In the case of the 6 September 2011 event, it was shown that the QSLs spread away from the inversion line, for magnetic models that included an unstable flux rope. Moreover, there were local changes similar to that found in the current density regions (investigated from HMI data during the same event) and the flare ribbons seen in the different filters of AIA.

As such, with different methods (magnetic field reconstruction and evolution, HMI and AIA), the authors were able to find the same consistent morphologies and evolution in flare ribbons, QSLs and electric current densities, as predicted in analytical (morphology) and numerical models (morphology and evolution). It is quite interesting that the standard flare model in three dimensions, derived from MHD simulations, is able to reproduce quite well the localisation of the impact of energetic particles as seen as flare ribbons, although it actually does not solve the 


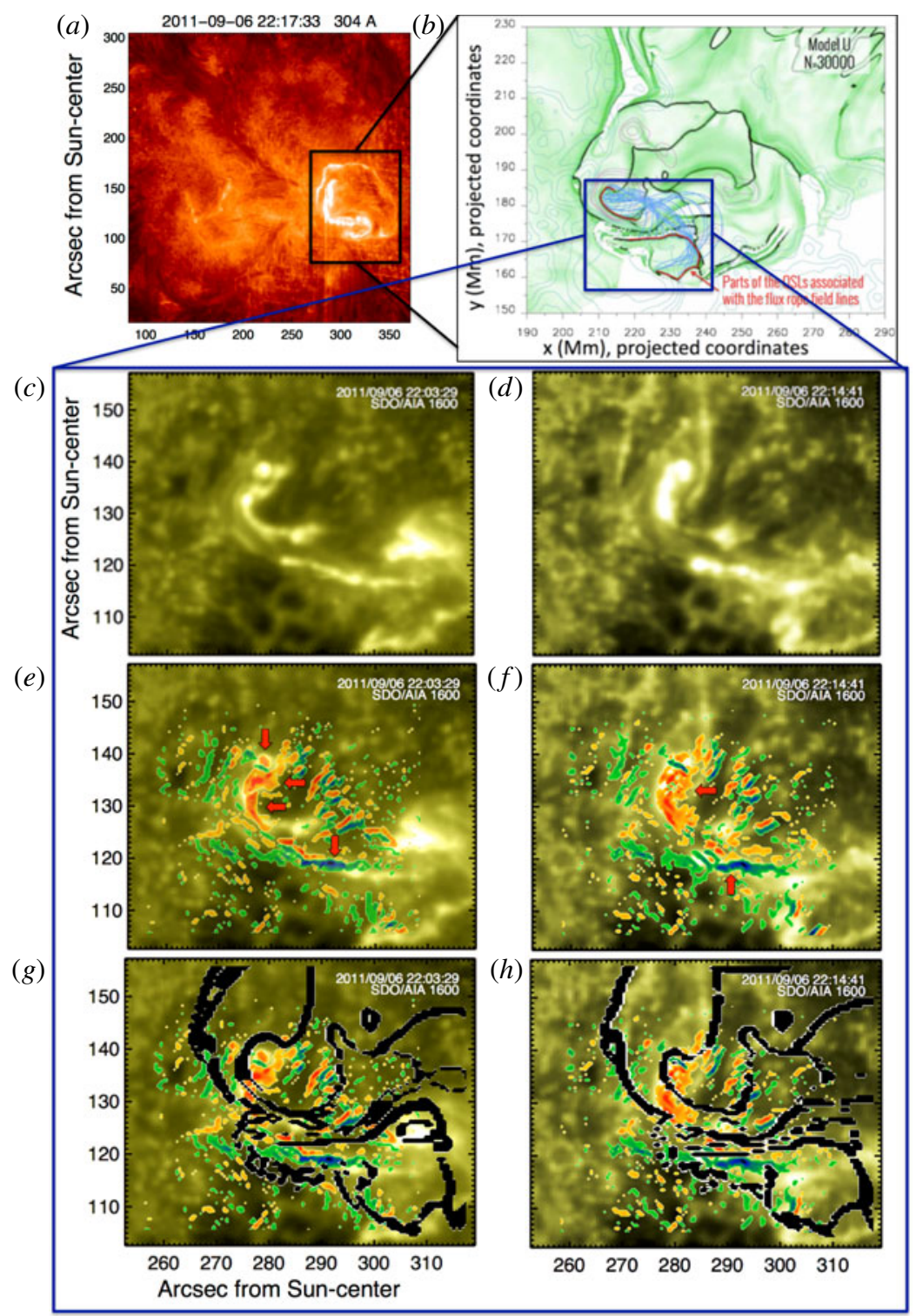

FIGURE 12. Current ribbons and QSL comparison in a complex flaring region. (a) Overview of the X-class flare region of 6 September 2011 in the $304 \AA$ channel of AIA aboard SDO. A large-scale circling flare ribbon (rectangle box) indicates the presence of a fan-like structure, while the most southern ribbon displays a hook shape typical of flux-rope ribbons. (b) QSL photospheric map of the zoomed region (black box in $a$ ) showing similar structures as the flare ribbons. In particular, a flux rope found in the extrapolated magnetic field (blue lines) is anchored in QSL regions displaying the typical $J$ shapes on both sides of the inversion line. A zoom on the two flare ribbons associated with the flux ropes are shown in the $1600 \AA$ filter before $(c)$ and after $(d)$ the impulsive phase. (e,f) An overlay of the same region with the current density obtained with the HMI data is shown for the same times. $(g, h)$ Same overlays adding the local QSLs from the extrapolation, showing a good agreement in the shape and the location of the QSLs (extrapolation), currents (HMI) and EUV flare ribbons. Adapted from Janvier et al. (2016). 

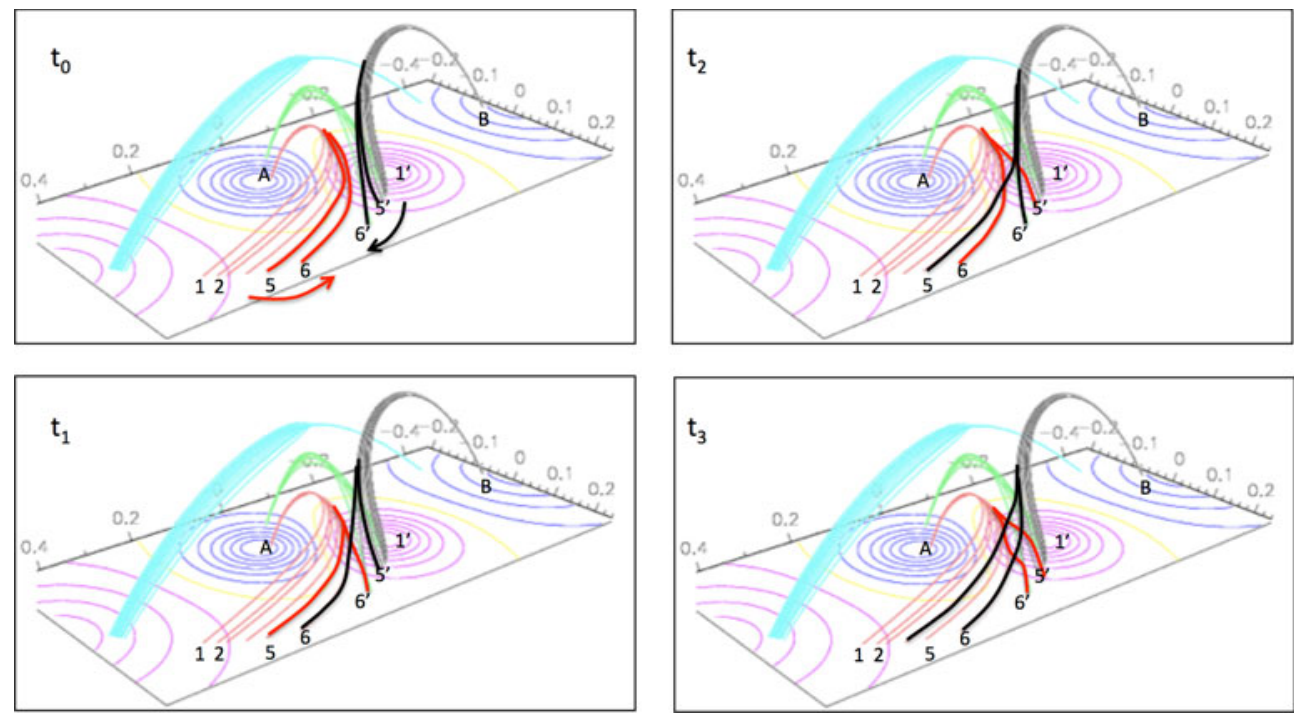

FIGURE 13. Representation of slipping field lines at different times in a numerical simulation. Four sets of field lines are represented, all defined from the negative polarity. The neighbouring anchorpoints of the cyan and black (respectively red and green) field lines in the same polarity, and the diverging locations of the corresponding footpoints in the positive polarity show that the connectivity remains continuous, while strongly diverging. The four thick red and black lines are defined as departing from local area A (red lines A5 and A6) and B (black lines B5' and B6'). At four different times, we look at the changes in the connectivity while those field lines are reconnecting with each other. The continuous change of connectivity gives an apparent slipping motion, indicated with the coloured arrows at $t_{0}$. (Adapted from Aulanier et al. (2006), see online for supplementary material showing the slipping motion in an animated gif.)

transport of energy to the lower layers of the Sun's atmosphere. Then, even though one can argue that MHD models do not take into account this transport (e.g. with energetic particles or waves), the fact that the evolution of the large-scale field is able to explain the observed features means that the MHD assumption is a strong and valid assumption to explain the large-scale features of the Sun's atmosphere.

\section{The consequences of 3-D reconnection in solar flares}

\subsection{Slipping reconnection}

Reconnection without a null point but in the presence of a parallel electric field due to the stress of magnetic fields near regions of strong distortions of the magnetic connectivities, was generalised in a series of papers and applied to the understanding of solar flares (see §2.2). In such a case, magnetic field lines are also seen to flip (Priest \& Forbes 1992) or slip (Priest \& Démoulin 1995), as they undergo a continuous change of connectivity in the reconnection layer.

To illustrate the notion of slipping field lines, let us take a set of field lines in figure 13 leaving the photospheric surface at similar locations: the red field lines leave from a footpoint neighbourhood $\mathrm{A}$ and the black lines a footpoint neighbourhood $\mathrm{B}$. In the following, we detail a connectivity change between two field lines at each time step, however note that this is only for a description convenience, since all field 
lines passing through the current volume would undergo continuous reconnection (see e.g. Priest, Hornig \& Pontin 2003). Note that the configuration is similar to figure 6, where the photospheric traces of the QSLs are shown in figure $6(b)$. We pick two field lines represented in thick lines, red A5 and A6 and black B5' and B6' (where the digits indicate the other footpoints). Note that the connectivity between the two positive magnetic polarities (represented in magenta) is continuous, although drastically changing as can be seen by the jump between 6 and $6^{\prime}$. Then, at the onset of reconnection, line A6 reconnects with its neighbouring field $B 6^{\prime}$ : at time $\mathrm{t} 1$, we can then define lines $\mathrm{A} 6^{\prime}$ and B6. As reconnection continues, we then have at $t_{2}$ newly formed $\mathrm{A} 5^{\prime}$ and $\mathrm{A} 6$, as well as $\mathrm{B} 5$ and $\mathrm{B} 6^{\prime}$. Reconnecting field lines then give an apparent sliding motion: the field line defined at $t_{0}$ as A5 has become A6 at $t_{2}$, then $\mathrm{A}^{\prime}$ at $t_{3}$, so that its anchoring footpoint in the positive polarity has an apparent motion going from points 5, 6 then $6^{\prime}$. It is the successive reconnections that is named the 'slipping' apparent motion of field lines.

At any time during the evolution of the magnetic field, one can define a specific field line by fixing one footpoint at the photospheric surface. More generally, in a numerical simulation where the bottom boundary evolves, one can define a field line by following its footpoint motion. That is the case for the yellow line shown in figure 14(a). We fix one of its footpoint in the negative polarity and at any time step in the simulation, field integration gives the location of the corresponding footpoint in the positive polarity.

If this field line is reconnecting, its change of connectivity can be followed with time: the corresponding footpoint location can be reported on a map such as in figure $14(b)$. Note that the field line is not jumping from one photospheric location to another: rather, the footpoint is continuously changing of locations because of the continuous change of connectivity (as there is no separatrices). Knowing the distances between two connectivity changes and the time step, one can then define a local slipping speed $v_{\text {slip }}$ : its variations during the reconnecting time interval are shown in figure $14(c)$.

Interestingly, the slipping speed (or connectivity change rate) is evolving in time. When compared with the Alfvén speed, we remark that the ratio can either be less than 1 or much larger: in Aulanier et al. (2006), the authors defined a sub-Alfvénic slipping motion as a motion for which the slipping speed is less than the Alfvén speed, and inversely for a super-Alfvénic motion. In the latter case, the slipping motion is referred to as a slip-running motion. In the example of figure 14 , the chosen field line slipping speed evolves from 0 to a sub-Alvénic speed, then a super-Alvénic speed. This actually represents the crossing of a QSL: further away from the QSL (and the current layer), the field line is not reconnecting $\left(v_{\text {slip }}=0\right)$. As the field line crosses the QSL, it undergoes reconnection with its neighbouring field lines (such as in figure 13). As the connectivity gradient increases while passing through the QSL, the field line reconnects with a neighbouring one which footpoint is even further away. This is seen as a larger 'jump' in the newly reconnected field line footpoint location, and a greater value of its slipping speed. In the core of the QSL, where the gradient is the greatest, the slipping speed is the largest, corresponding to a slip-running reconnection regime. As the field line is now exiting the QSL region, its slipping speed drops to sub-Alfvénic values, until reaching 0 again.

What dictates the slipping motion of field lines? The connectivity gradient is an important factor (and as such, is described by the geometry of the field, or QSLs), but so is the dissipation process within the current layer: as explained above, when passing through the dissipation volume, magnetic field lines reconnect with a 
(a)

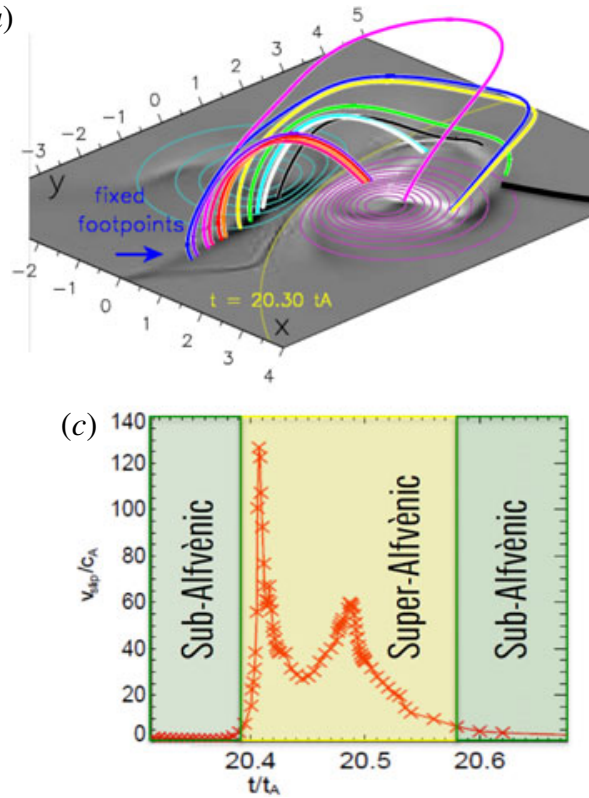

(b)

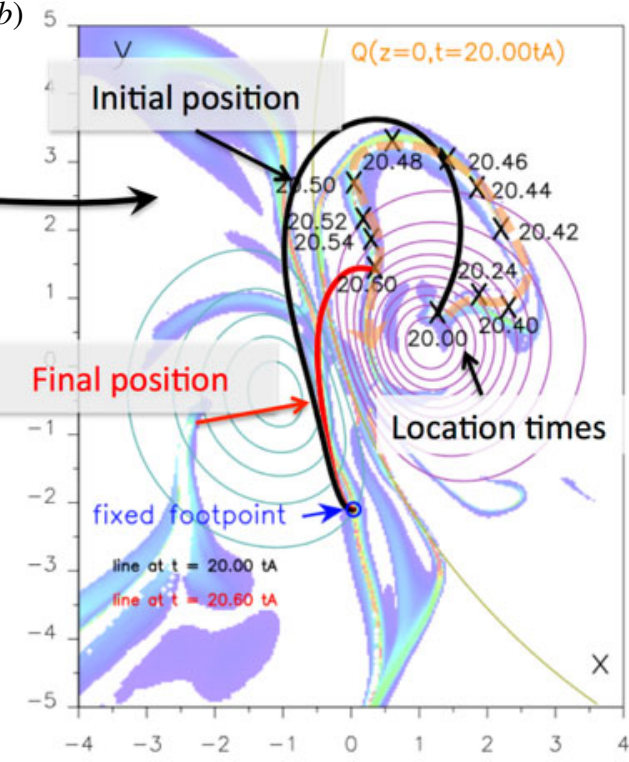

FIgURE 14. Calculation of the slipping speed. (a) Set of slipping field lines at a given time from an eruptive flare simulation (see Janvier et al. 2013), which anchoring point is indicated as fixed in the negative polarity. $(b)$ The footpoint locations of the moving yellow field line of $(a)$ at different times in the simulation are indicated by crosses, on an overlay of the QSL photospheric map. The initial position of the yellow field line is indicated as a black line, while its final position (when reconnection ends) is indicated as a red line (different colour coding as $(a)$ ). The path taken by the moving footpoint is indicated with an orange dashed line. $(c)$ Time evolution of the local speed of the moving footpoint (normalised by the Alfvén speed). Two regions are indicated. In green, we find the times when the motion is sub-Alfvénic $\left(v_{\text {slip }} \leqslant c_{A}\right)$, i.e. at the beginning and at the end of the QSL crossing. In yellow, we find the times when the motion is super-Alfvénic $\left(v_{\text {slip }} \geqslant c_{A}\right.$, slip-running motion, i.e. in the core region of the QSL where the connectivity gradient is the highest).

nearby field. Successive reconnections are then dictated by two aspects: the physical dissipation term which appears in Ohm's law (e.g. the resistivity) and the geometry, which provides an information on the connectivity of the neighbouring field with which field lines are going to reconnect with. This latter aspect was studied in great detail by Janvier et al. (2013), who found that the field line mapping norm (2.3) is essential in the speed of the slipping motion. Indeed, since slipping field lines are defined from one of the two footpoints, their slipping apparent motion will depend on the connectivity of their neighbouring field lines, which is given by the mapping norm. In other terms, the time evolution of the slipping speed, shown in figure $14(c)$, has the same profile as the spatial distribution of the mapping norm $N$ :

$$
v_{\text {slip }}=\alpha N
$$

where $v_{\text {slip }}$ represents the apparent speed of the moving footpoint and $N$ the mapping norm. The parameter $\alpha$ is itself a coefficient related with the reconnection rate within the current layer. Note that again, reconnection in the presence of QSLs generalises 
(a)

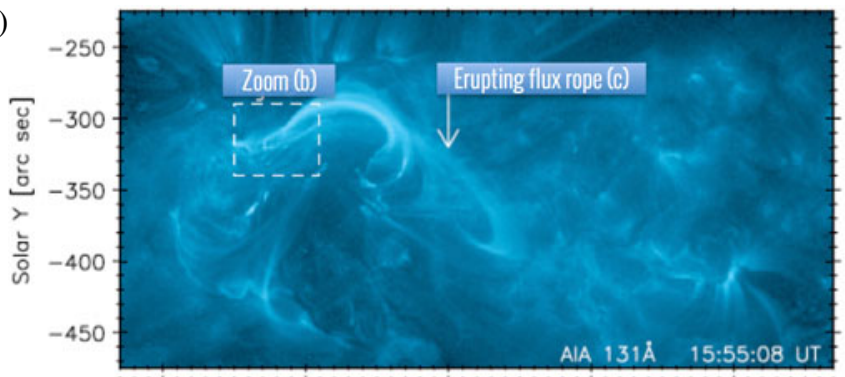

(b)

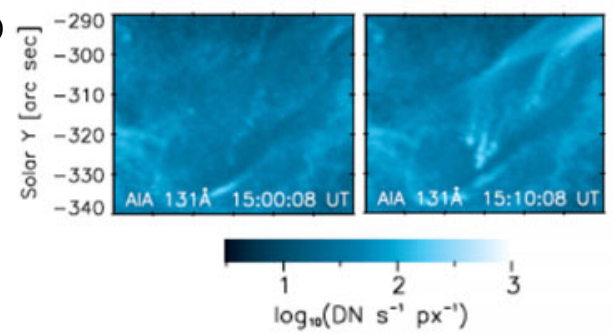

(c)
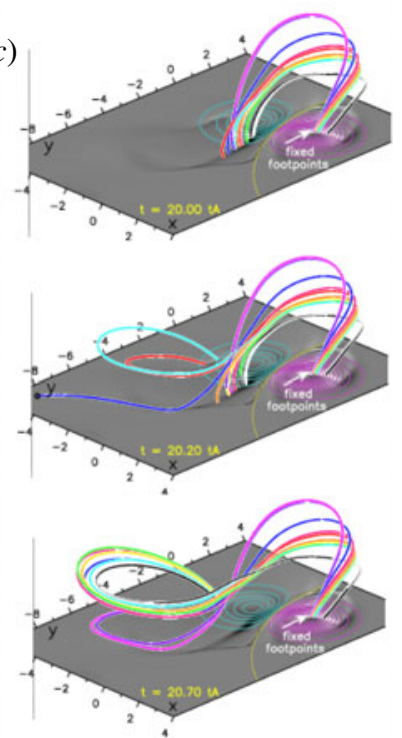

FIGURE 15. Evidence of apparent slipping motion during the X-class eruptive flare of 12 July 2012 (SOL2012-07-12T16:49). (a) Overview of the region where hot loops are seen in the $131 \AA$ (Fe XIII and Fe XXI), including an eruptive set of expanding loops (see figure 5 in Dudík et al. (2014)). The zoom region is shown in (b) at earlier times, where coherent, unidirectional motions of kernel brightening and apparent coronal loop motions are seen. (c) The analogy can be made with the slipping motion of magnetic field lines from a numerical simulation of an eruptive flares, where at different times reconnecting flux-rope field lines are seen to slip (adapted from Dudík et al. (2014)).

the results found in two dimensions: when the squashing degree $Q$ becomes infinite, in which cases QSLs generate real separatrices (in the topological sense), the slipping speed becomes infinite (the field line footpoint only 'moves' from an initial point to a final point) as $N=\infty$.

So far, we have only discussed slipping reconnection in terms of magnetic connectivity changes. However, these changes have consequences on the surrounding plasma. In the dissipation layer, the magnetic field energy is converted in other forms of energy such as kinetic energy of particles (later seen as Hard X-ray signatures) and heat. The apparition of bright kernels and flare ribbons at chromospheric altitudes during flares are understood as a transport of energy from the reconnection site in the corona to the chromosphere (see e.g. Graham \& Cauzzi 2015). Whether this energy is transported along field lines under the form of high energy particles thermal front or waves is still debated (Kerr et al. 2016). Since in the presence of QSLs, and as seen above, the change of connectivity is continuous and leads to a slippage of field lines, the apparently moving magnetic footpoint shown in figure 14 may have consequences on the sequential timing of the disturbances seen at the chromosphere.

With the high temporal cadence instrument AIA aboard Solar Dynamics Observatory, Dudík et al. (2014) were able to look in details at the motion of kernels seen during an eruptive flare. For the first time, a detailed analysis of these kernels at the highest time cadence of the instrument (12 s) showed that their motions are coherent: the kernels are seen to lit up successively in a privileged direction (figure 15). They are seen in different parts of the two ribbons appearing during the eruptive flare and 
accompanied by an apparent slipping motion of coronal loops (which was already pointed out in Aulanier et al. 2007, with X-ray observations of Hinode).

It may seem surprising that similar consequences are seen in both numerical simulations and observations. However, let us not forget that the plasma in the photosphere is at least $10^{9}$ times denser than the corona: disturbances from the corona to the photosphere would have great difficulties to affect and move the extremely massive plasma of the photosphere. As such, the field lines that would map the magnetic field from the photosphere can be said to be 'lined-tied' to the photospheric surface: hence, a similar interpretation as the simulation can be made.

Recently, several papers have reported similar analyses during eruptive flares showing the motion of kernels ( $\mathrm{Li} \&$ Zhang 2014; Li et al. 2016; Sobotka et al. 2016; Zheng, Chen \& Wang 2016). This should not be confused with the 'zipper'-propagation seen during prominence eruptions such as in Tripathi, Isobe \& Mason (2006): there, the locations of brightening kernels are seen to be related with the eruption direction of the large magnetic structure. Depending on whether the prominence lifts off symmetrically or asymmetrically, the flare kernels/ribbons appear sequentially at different locations due to the propagation of the reconnection site. With the intrinsic slipping motion of coronal loops, we can therefore expect to see kernel brightening appearing at the footpoints of both the erupting flux rope and the flare loops. This was reported in Dudík et al. (2016), where the authors showed observations of moving kernels belonging to both flare loops and the expanding flux rope.

Although kernels are related with high energy particles that can travel much faster than the bulk plasma, the plasma in the corona can also respond to the successive change of connectivity. Whether it is heating directly from the dissipation layer, or socalled evaporation from the chromosphere, newly formed coronal loops can be filled with hot plasma that is emitting in EUV and X-rays. However, the fastest speed at which the information of the change of connectivity can travel, in the plasma, is bound by the Alfvén speed: then, the apparent motion of field lines can be seen in observations when the slippage is sub-Alfvénic.

\subsection{Flare loops and flux rope}

Coronal loops in the Sun's atmosphere provide direct evidence of the consequences of magnetic reconnection during flares. They can be seen as strongly emitting features within the soft X-ray and EUV ranges. They typically appear after the onset of the flare, and can be seen throughout the impulsive phase and the decaying phase of the flare. These flare loops are seen in both confined and eruptive flares (Svestka 1986). In the latter case, flare loops typically form at higher altitude as time passes by, as the energy release site moves upward (Liu et al. 2004; Warren et al. 2011). Time sequences of flares also indicate that flare loops formed at the beginning of the flare have a more pronounced shear, evidenced by comparing their direction with that of the local polarity inversion line (PIL), than those formed later (Asai et al. 2003; Su et al. 2007). In Aulanier et al. (2012), the authors investigated the evolution of the shear from strong values to weak ones by comparing observations with a numerical model, and showed that coronal loops reconnecting later in time were also closer to potential loops, due to their initial low shear as well as to the dynamics of the ejected flux rope that decreases the shear of reconnecting field lines (see figure 16 which illustrates this dynamics). The flare ribbons lie at the feet of those dense loops. We often refer to an outward motion of the flare ribbons, away from the polarity inversion line, however, 
(a) Reconnecting pairs

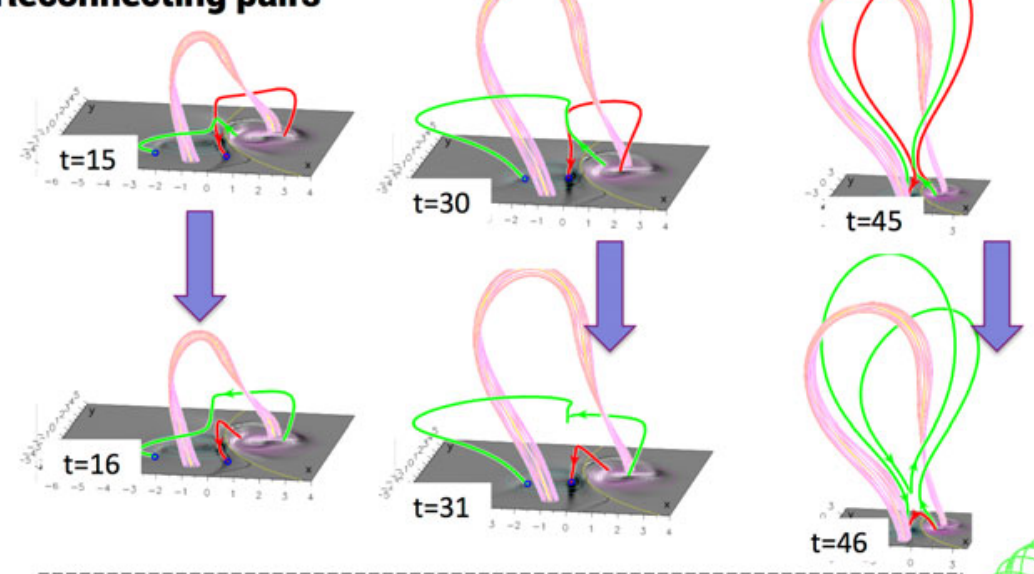

(b) Flux rope
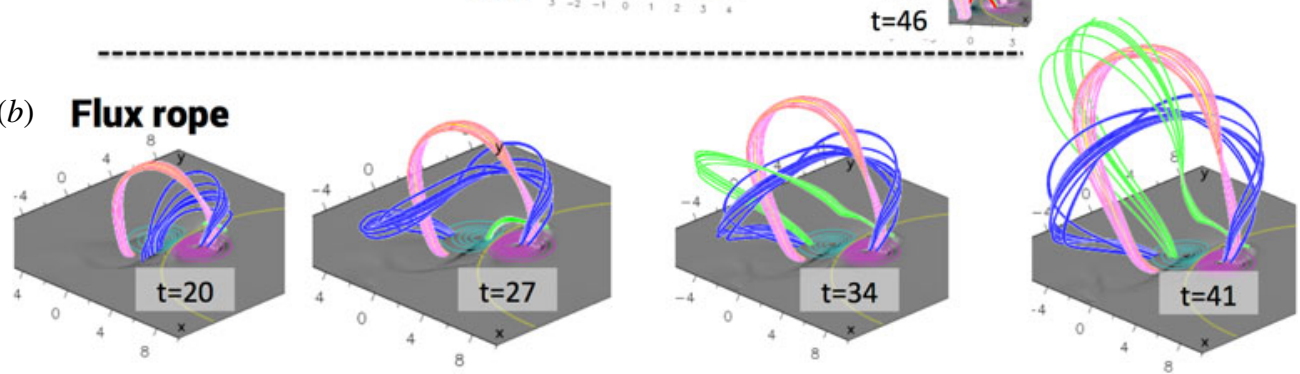

FIGURE 16. Consequences of reconnecting pairs of field lines. ( $a$, first row) Two pairs of field lines (red and green) chosen at different times and reconnecting with each other. ( $a$, second row) One Alfvén time later, newly reconnected field lines are the flare loop (in red) and a green field line that surrounds the flux rope (its core is represented in pink). As time goes by, the flux-rope field line forming on the outside becomes more twisted (e.g. at times 45-46 $t_{A}$ ) and the flare loop is less sheared. (b) Time evolution of selected neighbouring field lines in blue and green that undergo reconnection. The blue field lines reconnect earlier than the green ones, and both form the successive layers of the flux-rope envelope. Adapted from Aulanier et al. (2012).

this motion is only apparent as it is related with the progressive lighting up of the regions where newly reconnected flare loops are anchored, as reconnection propagates.

In eruptive flaring active regions, evidence of an already present twisted structure can be found. This is seen as the presence of a hot bundle of $S$ - or $J$-shaped loops referred to as a sigmoid, which are generally emitting in hard X-rays (Gibson \& Fan 2006; Green et al. 2007; Green \& Kliem 2009; Tripathi et al. 2009). In a series of papers, Savcheva \& van Ballegooijen (2009) and Savcheva et al. (2012a,b, 2014) showed that sigmoids are recurrent in flaring active regions. This was also investigated by Nindos et al. (2015), by using different AIA channels (131, 171 and $304 \AA$ ) who concluded that flux ropes and flux-rope-like structures can be seen prior to eruptions in $30 \%-50 \%$ of the cases. Such studies clarify the existence of flux ropes before the eruption, although direct observations are difficult, and hence the presence of a flux rope cannot be verified directly until accessing quantitative measurements of magnetic fields in the corona. The existence of flux ropes prior to flares is corroborated with findings of numerical simulations. Photospheric motions 
such as diffusion and flux cancellation permit creation of erupting flux ropes, as was modelled in 3-D simulations (Amari et al. 2003a; Aulanier et al. 2010). The study of the destabilisation of flux ropes, at the origin of eruptive flares, is out of scope of the present paper, and we refer the reader to the review of Schmieder, Démoulin \& Aulanier (2013).

Upon the eruption, these structures can be observed in coronagraphic observations as a three-part structure (e.g. Cremades \& Bothmer 2004) but also more recently in low coronal observations. They are observed off limb as large structures with a cavity (Gibson et al. 2010; Aparna \& Tripathi 2016). Thanks to numerical simulations, it is possible to probe into the evolution of flux ropes. As the magnetic field of the active region undergoes reconnection, creating flare loops, the counterpart of this reconnection process is the formation of the flux-rope envelope: the reconnected field forms field lines that are wrapping around the core of the flux rope. This is shown in figure 16, where pairs of field lines are shown to end up on the one hand as flare loops and on the other hand as part of the flux-rope envelope. Those field lines then become part of the flux rope, and the latter grows in size (figure 16b). As such, the flux rope is always in constant magnetic flux evolution during the flare. This evolution can also be seen by studying the dimming regions, generally associated with the footpoint of the flux rope (see Webb \& Howard 2012, and references therein). In particular, the evolution of these regions gives a good indication of the flux content in flux ropes (Qiu et al. 2007). In some cases, those dimming regions are also seen to have a strong-to-weak shear evolution similarly to the flare loops, as is also shown in simulations such as in figure 16 (Miklenic et al. 2011). Also, the flux rope that is seen to evolve in the Sun's corona can be linked with the interplanetary medium, where direct in situ observations give us a quantification of the flux and the rotation of the magnetic field, that can then be used to find the properties, such as the morphology of the flux rope, its flux and twist content (Dasso et al. 2005; Mandrini et al. 2005; Hu et al. 2014).

\subsection{Plasmoids and outflows}

Numerical simulations of magnetic reconnection have shown that the evolution of unstable current layers (such as the tearing mode) leads to the formation of magnetic islands in two dimensions or plasmoids, that can be understood as small flux ropes in three dimensions. They are represented in two dimensions by nested magnetic field lines (see figure 17), while in three dimensions it is much more difficult to define what a plasmoid actually is. Indeed, in three dimensions, what would look like a coherent entanglement of field lines in one location may not be actually so different from the surrounding magnetic field in other locations in the 3-D volume, as discussed in $\S 3.3$ (see for example the magnetic field rendering of the 3-D simulation in Daughton et al. 2011; Nishida et al. 2013). Recent numerical simulations such as by Baalrud, Bhattacharjee \& Huang (2012), Wyper \& Pontin (2014) provide invaluable tools to comprehend the link between 2-D and 3-D plasmoid dynamics.

The formation and the evolution of plasmoids were invoked as an essential component in the impulsive, fast reconnection regime encountered in the solar corona: as a plasmoid is formed, the current layers become thinner, leading to a new Sweet-Parker regime that can become unstable to a secondary instability (Shibata \& Tanuma 2001). As explained in Edmondson et al. (2010) and Lynch et al. (2016), the plasmoid formation is a robust and universal process found in varied plasma simulations. It ranges from MHD to more detailed simulations taking into account 
(a)

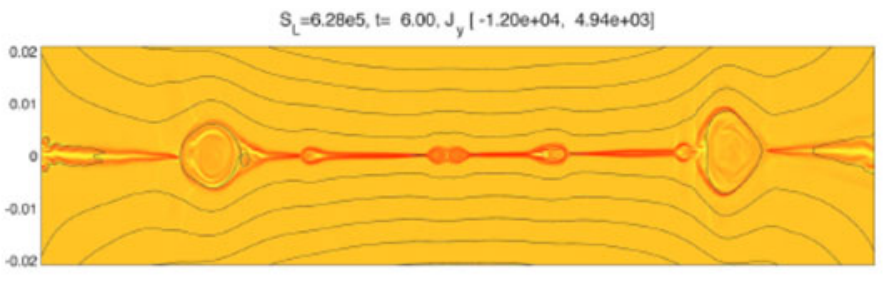

$\mathrm{S}_{\mathrm{L}}=6.28 \mathrm{e}, \mathrm{t}=9.10, \mathrm{~J}_{\mathrm{y}}[-9.59 \mathrm{e}+03,3.71 \mathrm{e}+03]$

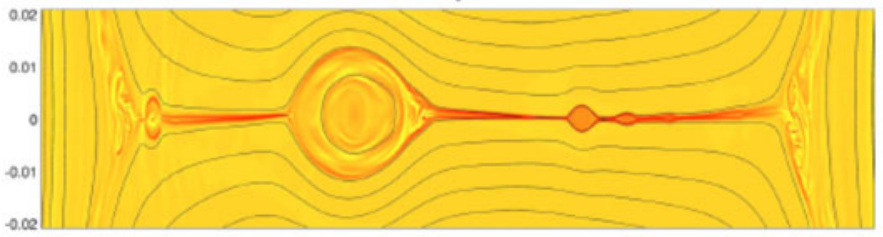

$S_{L}=6.2805, t=12.00, J_{y}[-9.110+03,4.700+03]$

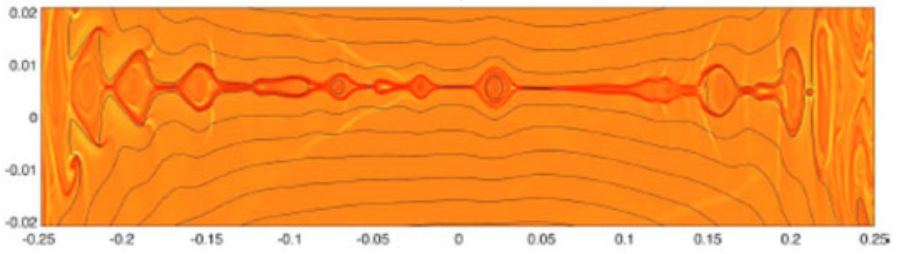

(b)

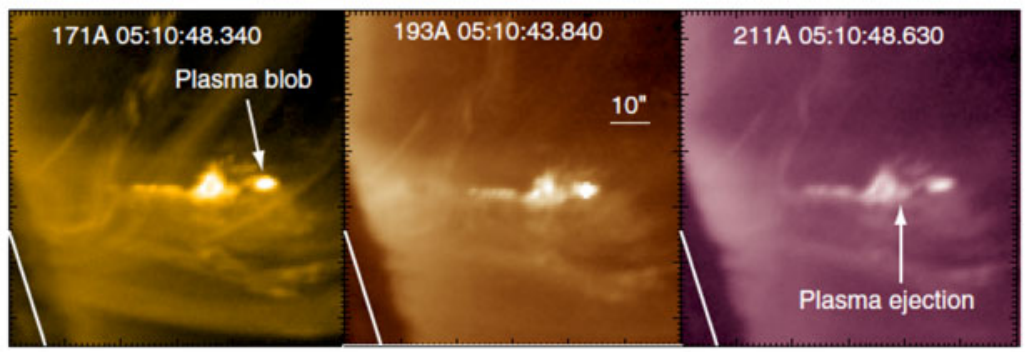

(c)

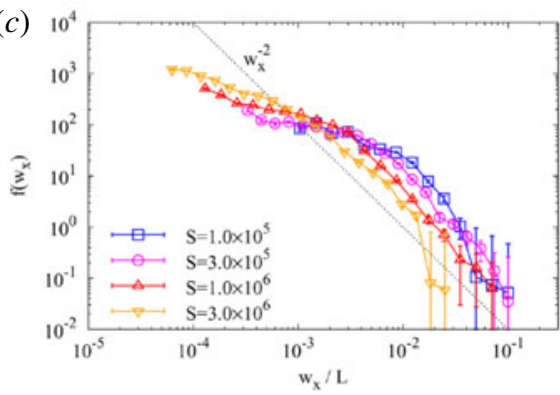

(d) $\log _{10}\left(\mathrm{~d} N_{o b s} / \mathrm{d} R\right)$

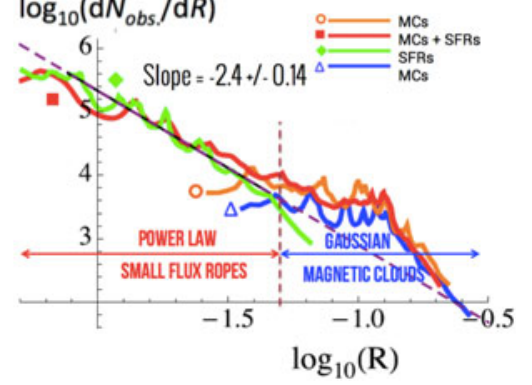

FIGURE 17. Plasmoids in numerical simulations and observations. (a) Numerical simulations of Bhattacharjee et al. (2009), where the formation and coalescence of plasmoids can be seen, creating large magnetic islands. (b) Remote sensing observations of the Sun's corona during an eruption, where plasma blobs are seen during the rising phase of a flare and above the flare loop top (adapted from Takasao et al. (2012)). (c) Power law found in the distribution of the size of plasmoids in computer simulations, from Loureiro et al. (2012). (d) A similar power law is found for small flux ropes directly observed in the interplanetary medium, from the study of Janvier, Démoulin \& Dasso (2014b). 
particle effects. As they explain, inflows and outflows are determined by the global geometry of the system (which dictates the geometry of the current sheet, i.e. the region where the frozen-in condition breaks down) and the resistivity/particle effect modelled (which dictates the diffusion scale). Since the system is also determined with other constraints such as conservation of mass and magnetic flux, the creation of magnetic islands and the further tearing of current sheets, such as in the plasmoid instability scenario, allows the system to introduce new scales to accommodate both the global constraints and conservation laws.

This subsequent nonlinear evolution from a tearing unstable current layer has since then been investigated in many different simulations. They indeed show the nonlinear evolution of unstable current layers into a Sweet-Parker regime and a further secondary plasmoids creation regime (e.g. Loureiro et al. 2005; Samtaney et al. 2009; Ni et al. 2010; Militello, Grasso \& Borgogno 2014). This was also confirmed in laboratory experiments by Liang et al. (2007). Because plasmoids can grow, either from the subsequent evolution of the instability (continued reconnected flux accumulation) or by coalescence of magnetic islands (Hayashi 1981), large plasmoids can be generated. Then, it is possible to study statistically the occurrence of plasmoids from different sizes (see e.g. the simulation of Bhattacharjee et al. (2009) in figure 17a). In particular, Fermo, Drake \& Swisdak (2010), Uzdensky, Loureiro \& Schekochihin (2010) and Loureiro et al. (2012) discuss the formation of large plasmoids (also coined 'monster' plasmoids by the latter two), which were also found by Lynch et al. (2016) in simulations of current sheets during eruptive flares. Such simulations are very interesting for comparisons with direct or indirect observations of plasmoids, both in the Sun's atmosphere and the heliosphere.

The main consequences of magnetic reconnection are directly seen in remote sensing observations as a large change in bolometric measurements (i.e. light curves), ranging from microwaves to X-rays and even gamma rays. Then restructuring of the magnetic field as seen above, as well as outflows of structures such as plasmoids, have also been reported. For example, Yokoyama et al. (2001) reported reconnection inflows in remote sensing observations of a limb flare event, while recently Takasao et al. (2012) and Liu, Chen \& Petrosian (2013) reported clear observations of plasmoids in the trailing region of an erupting prominence, and in the region above the flare loops (see figure 17b). Other consequences of magnetic reconnection in coronal current layers after the onset of an eruption, such as turbulence (Bemporad 2008) and supra-arcade downflows (see e.g. McKenzie 2011; Savage et al. 2012a; Savage, McKenzie \& Reeves 2012b), as well as hot high-speed plasma outflows (Wang, Sui \& Qiu 2007) have been reported over the years. Note that the motion of plasmoids (or plasma blobs in the corona) jetted away from the reconnection site (either upward or downward) is also reproduced in numerical simulations (e.g. Bárta, Vršnak \& Karlický 2008).

Radio observations also show drifting pulsating structures (DPS). These radio emissions differ from type II or type III bursts as they appear as intermittent bursts, and are believed to originate from the trapping of electrons inside plasmoids (e.g. Karlický, Fárník \& Mészárosová 2002). Then, as these particles travel inside the structure, their radio emissions are directly related with the density and the size of the structure in which they are trapped. Indeed, analyses of DPS in radio emissions and remote sensing images of plasmoids taken for example by AIA show a good correspondence in their occurrences and locations (Nishizuka et al. 2015). Nishizuka et al. (2015) also found a distribution of plasmoid width centred around $6 \times 10^{8} \mathrm{~cm}$. According to Loureiro et al. (2012), the maximum plasmoid size found in numerical 
simulations scaled as a tenth of the system size. Then, with plasmoids of the order of a megametre, one would expect a current layer of $0.1 \mathrm{Mm}$. This seems to fit the observations of Takasao et al. (2012) (see figure 17(b) and their paper for an observation of what would be the coronal current layer structure). We note that the correspondence between the plasmoids found in the corona, as indicated in the discussions above, and the plasmoids found in simulations, are yet to be confirmed. Indications may be given by the link between coronal plasmoids and small structures found in the interplanetary medium, as follows.

Finally, as flux ropes are ejected in the interplanetary medium, it is possible to directly probe them with in situ instruments (such as with STEREO A/B, Wind or ACE at $1 \mathrm{AU}$ ). Such data have been for example used to obtain diagnostics of the structures of coronal mass ejections (e.g. Zurbuchen \& Richardson 2006, and references therein). A population of small flux ropes have been found to have a similar magnetic structure as interplanetary CMEs (twisted magnetic field), while following a completely different size distribution (see Janvier et al. 2014b, and figure $17(d)$ ). Interestingly, the power law of the distribution of small flux ropes is similar to the one found in numerical simulations of plasmoids (figure 17c). This indicates that similar processes in the formation of those plasma structures may occur in the current layers of the Sun's atmosphere. This is an interesting finding that supports the universality of the reconnection phenomenon and its consequences, from plasma experiments to numerical simulations and astrophysical plasmas.

\section{Discussion and conclusion}

Magnetic reconnection is a very complex phenomenon, that can be studied from various angles: analytical, numerical, observational, to study the change in topology, the energy conversion, MHD processes, bifluid or (gyro-)kinetic ones, etc. The different approaches are so numerous it is of course impossible to report all the findings in every branch of reconnection studies.

In the present review, the interest is focused on presenting this variety, as a guideline so as to remember that magnetic reconnection is difficult to understand in its ensemble, yet its studies and the advances along the years have provided powerful insights in the physics of solar flares. Especially, the advances provided by an increasing power in numerical calculations, as well as high spatial and temporal observations, have made possible the understanding of the magnetic reconfiguration that ensues from the reconnection mechanism.

As such, we have reported in $\S 2$ how to understand magnetic reconnection from a topological point of view, looking at places where the field line connectivity is discontinuous (such as null points and separators, §2.1) or where the magnetic connectivities are strongly distorted ( $\$ 2.2)$, leading under stress to the formation of current layers $(\S 3)$ where the magnetic energy is dissipated. For researchers interested in the mechanisms of the dissipation process, multiple 'layers' can be considered: at MHD scales, the evolution of boundary motions or spontaneous instability lead to the formation of magnetic islands (in a 2-D cut), while at smaller scales, the reconnection rate is influenced by the behaviours of ions and electrons ( $\$ 3.1)$. At scales that are of interest to understand the process of eruptive flares, numerical studies give us some insights on the structure and the evolution of the 3-D current volume (§3.2), which can both be directly compared with observations (\$3.4). As such, the aim of the paper is to provide a thorough understanding of the evolution of the standard model for flares in three dimensions. 
The consequences of reconnection in three dimensions are multiple and have been reported in $\S 4$. We can first point out that the intrinsic 3-D nature of magnetic reconnection leads to phenomena such as slipping motion of field lines that have recently been confirmed by high temporal cadence observations of the Sun's atmosphere $(\$ 4.1)$. The magnetic field 3-D restructuring leads to typically observed evolution of flare loops and flux ropes. For example, the strong-to-weak shear transition of flare loops as well as the growing envelope of flux ropes $(\$ 4.2)$ are seen in both numerical studies and observations. Finally, observations at high spatial resolutions of the corona have revealed fine structures such as plasmoids. They have been the subject of many studies and provide comparisons between observations and numerical simulations of teared current layers $(\$ 4.3)$. There are however other aspects that have not been touched upon in the present review, for reasons specified below.

The consequences of magnetic reconnection are related with the restructuring of the magnetic field as well as with the dissipation of magnetic energy. Then, one question that derives from the mechanisms of solar flares is what proportion of free magnetic energy (i.e. the difference between non-potential magnetic field and potential magnetic field energies) is actually converted in other sources of energy, and how this partition occurs. Syrovatskii (1966) described that the electric field is directed along $\boldsymbol{J}$, and thus will perform positive work on the charged particles, increasing their energy. It is this process that will convert the magnetic energy into the kinetic energy of the particles (dynamic dissipation). Such a mechanism is distinct from Joule dissipation as there is not a simple proportionality between the current density and the electric field. In van Hoven \& Cross (1973), the authors discussed the energy release during the tearing instability, and showed that $10 \%$ of the background energy can be released under the form of non-thermal energy. More recently, Yamada, Yoo \& Zenitani (2016) showed that in both plasma experiments and 2-D simulations, approximately half of the magnetic energy is converted into particle energy, of which $2 / 3$ is ultimately transferred to ions and $1 / 3$ to electrons. Since those dedicated experiments and simulations are mainly focused on the dissipation layer, it would be interesting to extend such studies to their consequences in the large-scale changes seen during flares. For example, is there a link between the energy conversion seen at the dissipation scale, the energy carried by the energy spectrum of particles during flares/waves (for example, high-frequency Alfvén waves energy conversion in the presence of turbulent magnetic fields, as investigated by Lazarian \& Vishniac 1999) and the kinetic energy of the eruptive magnetic flux rope?

Bolometric energy (or total radiant energy) is a good proxy to infer the amount of energy that is released during flares under the form of direct heating and particle energy (emitting in, e.g. hard X-rays). However, obtaining its values for flares is very difficult (Kretzschmar 2011). From different flare events, Emslie et al. (2005) reported that (at least) $50 \%$ of the magnetic energy was converted into the bolometric flare energy while the other half in the kinetic energy of the CME. However, the estimation of the kinetic energy comes with a lot of hypotheses, such as the mass that is transported in CMEs. Such masses are generally inferred from remote sensing coronal observations (such as with the LASCO instrument suites aboard SoHO), but can be mixed with the dense region created by a snow-plow effect of the erupting CME which is generally defined as the sheath region, well observed with in-situ instruments. This was for example reported by Feng et al. (2015) where the authors found that the effect of the solar wind 'snow-plow' phenomenon was not negligible.

In numerical experiments, large-scale (either 2.5-D or 3-D) MHD simulations of eruptive flares such as in Amari et al. (2003b), Lynch et al. (2008), Reeves 
et al. (2010), Aulanier et al. (2012) show that the kinetic energy of the CME is approximately $5 \%$ and not more than $10 \%$, well below the amount proposed from observations. These discrepancies between numerical simulations and observations, as discussed by Aulanier et al. (2013), must be resolved in the future both by improving the numerical scheme (dissipation mechanisms and atmospheric models in simulations) and the hypotheses/data constraints (the actual plasma mass transported in CMEs).

Comments have been made that MHD simulations do not treat particles needed to explain the conversion of magnetic energy at ion and electron scales, as well as other nonlinear effects such as wave-particle interactions. However, as was shown throughout the text, the MHD paradigm is very good at explaining a varied range of observations. For example, we have seen that the strong gradients of the field line mapping are related to locations of strong current densities. These correspond very well with regions of heating and particle acceleration such as null points and QSLs. Some may argue that the use of numerical techniques (as well as analytical ones) constrain the analysed configurations: those are over-simplified, when compared with the complexity of active regions and flare configurations. Nonetheless, studying such simplified models is still valuable, because it provides a quantitative test of the laws of physics we use to explain the underlying mechanisms of flares.

Furthermore, studying the large-scale restructuring of the magnetic field at MHD scales let us understand how flux ropes are created and are ejected in the heliosphere, Then, studies focused on their helicity content and $B_{z}$ orientation (important to estimate the impact on magnetospheres) are made possible by understanding the whole sequence of MHD evolution. As pointed out by Forbes (2000), there is an enormous mathematical difficulty in solving the equations governing the motion of plasma and the behaviour of magnetic fields. One the one hand, fluid equations have a high degree of nonlinearity, well illustrated by turbulent behaviours, and on the other hand, numerical methods cannot handle well (yet) the large gap between scales seen in solar flares, from the electron gyro-radius at $10^{-2} \mathrm{~m}$ to the megametre range $\left(10^{9} \mathrm{~m}\right)$ observed in CMEs. A solution would be to bridge different simulations, for example by providing MHD solutions as inputs in energetic particles models describing the motion of the particles in electromagnetic fields.

In the GEM challenge (Geospace Environmental Modeling Magnetic Reconnection Challenge, see §3.1), the authors (Birn et al. 2001) found that different codes that include small-scale physics are different from the MHD code. In particular, all the models that include the Hall effect in the generalised Ohm's law behave similarly (in terms of reconnection rate), and with a higher reconnection rate than the MHD model. It looks like the effect of the Whistler waves, brought in to the dynamics by the Hall term, is important in modelling magnetic reconnection. How this will affect the evolution of the magnetic field during solar flares, in three dimensions, is something that is still to be analysed. Moreover, recent studies such as that of Bessho \& Bhattacharjee (2005) show even when the Hall term is not present (cancelled out, in the case of an electron-positron plasma), while fast reconnection still takes place, mediated by the pressure tensor (which off-diagonal terms produce an effect similar to spatially localised resistivity). Effects at electron scales therefore may provide invaluable insights in understanding the mechanisms solar flares. Finally, in full 3-D systems, current layers are unstable to a wider range of instabilities than in a 2-D system, so this is something that remains to be looked at more closely.

Another great difficulty in understanding the mechanisms of flares reside in its time scale: during the impulsive phase of large flares, which lasts a few minutes, 
approximately $10^{25} \mathrm{~J}$ of energy is released within a short amount of time. How can such amount of energy be converted into thermal and kinetic energy within the dissipation layer, which can be assumed to be of the order of $10^{15} \mathrm{~km}^{3}$ ?

Citing Gonzalez \& Parker (2016), the editors wisely wrote 'One can also see that no consensus about (fundamental issues in magnetic reconnection) is presently available, thus indicating that the subject of magnetic reconnection remains open for further theoretical, computational, and observational studies'. Nonetheless, reconnection during eruptive flares is an exciting field where progresses have allowed us to understand, but also predict its underlying mechanisms. Dedicated missions such as the future Solar Orbiter will allow to better constrain the physical inputs of reconnection consequences, such as flux-rope ejections, by directly probing the near environment of the corona.

\section{Acknowledgements}

The author would like to thank the two reviewers for their comments which help improve the manuscript. She would like to acknowledge the discussions she had at the International Scientific Seminar 'Structure and dynamics of the solar atmosphere: recent advances and future challenges', Kavli Royal Society International Center, which resulted in the addition of some materials for the present manuscript. The author would also like to thank T. Passot, editor of JPP, for his patience in receiving the manuscript, P. Démoulin for his comments helping the revision of the text and C. Damiani for her support.

\section{REFERENCES}

Al-Hachami, A. K. \& Pontin, D. I. 2010 Magnetic reconnection at 3d null points: effect of magnetic field asymmetry. Astron. Astrophys. 512, A84.

Amari, T., Luciani, J. F., Aly, J. J., Mikić, Z. \& Linker, J. $2003 a$ Coronal mass ejection: initiation, magnetic helicity, and flux ropes. I. Boundary motion-driven evolution. Astrophys. $J$. 585, 1073-1086.

Amari, T., Luciani, J. F., Aly, J. J., Mikić, Z. \& Linker, J. $2003 b$ Coronal mass ejection: initiation, magnetic helicity, and flux ropes. II. Turbulent diffusion-driven evolution. Astrophys. J. 595, 1231-1250.

Aparna, V. \& Tripathi, D. 2016 A hot flux rope observed by SDO/AIA. Astrophys. J. 819, 71.

Asai, A., Ishit, T. T., Kurokawa, H., Yokoyama, T. \& Shimojo, M. 2003 Evolution of conjugate footpoints inside flare ribbons during a great two-ribbon flare on 2001 April 10. Astrophys. J. 586, 624-629.

Aulanier, G., Deluca, E. E., Antiochos, S. K., McMullen, R. A. \& Golub, L. 2000 The topology and evolution of the bastille day flare. Astrophys. J. 540, 1126-1142.

Aulanier, G., Démoulin, P., Schrijver, C. J., Janvier, M., Pariat, E. \& Schmieder, B. 2013 The standard flare model in three dimensions. II. Upper limit on solar flare energy. Astron. Astrophys. 549, A66.

Aulanier, G., Golub, L., Deluca, E. E., Cirtain, J. W., Kano, R., Lundquist, L. L., NARUkage, N., SAKaO, T. \& Weber, M. A. 2007 Slipping magnetic reconnection in coronal loops. Science 318, 1588.

Aulanier, G., Janvier, M. \& Schmieder, B. 2012 The standard flare model in three dimensions. I. Strong-to-weak shear transition in post-flare loops. Astron. Astrophys. 543, A110.

Aulanier, G., Pariat, E. \& DÉmoulin, P. 2005 Current sheet formation in quasi-separatrix layers and hyperbolic flux tubes. Astron. Astrophys. 444, 961-976.

Aulanier, G., Pariat, E., Démoulin, P. \& DeVore, C. R. 2006 Slip-running reconnection in quasi-separatrix layers. Solar Phys. 238, 347-376. 
Aulanier, G., Török, T., Démoulin, P. \& Deluca, E. E. 2010 Formation of torus-unstable flux ropes and electric currents in erupting sigmoids. Astrophys. J. 708, 314-333.

BaAlrud, S. D., Bhattacharjee, A. \& HuAng, Y.-M. 2012 Reduced magnetohydrodynamic theory of oblique plasmoid instabilities. Phys. Plasmas 19 (2), 022101.

Bagalá, L. G., Mandrini, C. H., Rovira, M. G. \& Démoulin, P. 2000 Magnetic reconnection: a common origin for flares and AR interconnecting arcs. Astron. Astrophys. 363, 779-788.

BARnes, G., Longcope, D. W. \& LEKA, K. D. 2005 Implementing a magnetic charge topology model for solar active regions. Astrophys. J. 629, 561-571.

BÁrta, M., VRŠnaK, B. \& KARLICKÝ, M. 2008 Dynamics of plasmoids formed by the current sheet tearing. Astron. Astrophys. 477, 649-655.

Baty, H., Forbes, T. G. \& Priest, E. R. 2009 Petschek reconnection with a nonlocalized resistivity. Phys. Plasmas 16 (1), 012102.

BAty, H., Forbes, T. G. \& Priest, E. R. 2014 The formation and stability of Petschek reconnection. Phys. Plasmas 21 (11), 112111.

Baum, P. J. \& Bratenahl, A. 1976 Laboratory solar flare experiments. Solar Phys. 47, 331-343.

BAUM, P. J. \& BRATENAHL, A. 1980 Flux linkages of bipolar sunspot groups - a computer study. Solar Phys. 67, 245-258.

Baum, P. J., Bratenahl, A., Crockett, G. \& Kamin, G. 1979 Magnetic flux of colinear bipolar SPOT pairs. Solar Phys. 62, 53-67.

Baum, P. J., Bratenahl, A., Kao, M. \& White, R. S. 1973 Plasma instability at an X-type magnetic neutral point. Phys. Fluids 16, 1501-1504.

BEMPorad, A. 2008 Spectroscopic detection of turbulence in post-CME current sheets. Astrophys. J. 689, 572-584.

Bessho, N. \& BhattacharJee, A. 2005 Collisionless reconnection in an electron-positron plasma. Phys. Rev. Lett. 95 (24), 245001.

Bhattacharjee, A., Huang, Y.-M., Yang, H. \& Rogers, B. 2009 Fast reconnection in highLundquist-number plasmas due to the plasmoid Instability. Phys. Plasmas 16 (11), 112102.

Bierwage, A., Hamaguchi, S., Wakatani, M., Benkadda, S. \& Leoncini, X. 2005 Nonlinear evolution of $q=1$ triple tearing modes in a tokamak plasma. Phys. Rev. Lett. 94 (6), 065001.

Birn, J., Drake, J. F., Shay, M. A., Rogers, B. N., Denton, R. E., Hesse, M., Kuznetsova, M., Ma, Z. W., Bhattacharjee, A., Otto, A. et al. 2001 Geospace environmental modeling (GEM) magnetic reconnection challenge. J. Geophys. Res. 106, 3715-3720.

BIRn, J.\& Hesse, M. 2001 Geospace environment modeling (GEM) magnetic reconnection challenge: resistive tearing, anisotropic pressure and Hall effects. J. Geophys. Res. 106, 3737-3750.

BISKAMP, D. 1986 Magnetic reconnection via current sheets. Phys. Fluids 29, 1520-1531.

Bommier, V., Landi Degl'Innocenti, E., LAndolfi, M. \& Molodiu, G. 2007 UNNOFIT inversion of spectro-polarimetric maps observed with THEMIS. Astron. Astrophys. 464, 323-339.

BÜChNeR, J. 2007 Astrophysical reconnection and collisionless dissipation. Plasma Phys. Control. Fusion 49, B325-B339.

CAI, H. \& LI, D. 2008 Magnetic reconnection with electron viscosity in electron magnetohydrodynamics. Phys. Plasmas 15 (3), 032301.

Carmichael, H. 1964 A process for flares. NASA Special Publication 50, 451.

Chandra, R., Schmieder, B., Aulanier, G. \& Malherbe, J. M. 2009 Evidence of magnetic helicity in emerging flux and associated flare. Solar Phys. 258, 53-67.

Chandra, R., Schmieder, B., Mandrini, C. H., Démoulin, P., Pariat, E., TöröK, T. \& UDDIN, W. 2011 Homologous flares and magnetic field topology in active region NOAA 10501 on 20 November 2003. Solar Phys. 269, 83-104.

Comisso, L. \& Bhattacharjee, A. 2016 On the value of the reconnection rate. Preprint.

Cowling, T. G. 1945 On the Sun's general magnetic field. Mon. Not. R. Astron. Soc. 105, 166.

CRAig, I. J. D. \& EFFEnBERGer, F. 2014 Current singularities at quasi-separatrix layers and three-dimensional magnetic nulls. Astrophys. J. 795, 129.

Cremades, H. \& Bothmer, V. 2004 On the three-dimensional configuration of coronal mass ejections. Astron. Astrophys. 422, 307-322. 
Dasso, S., Mandrini, C. H., Démoulin, P., Luoni, M. L. \& Gulisano, A. M. 2005 Large scale MHD properties of interplanetary magnetic clouds. Adv. Space Res. 35, 711-724.

Daughton, W., Roytershteyn, V., Karimabadi, H., Yin, L., Albright, B. J., Bergen, B. \& BOWERS, K. J. 2011 Role of electron physics in the development of turbulent magnetic reconnection in collisionless plasmas. Nat. Phys. 7, 539-542.

De La Beaujardiere, J.-F., CAnfield, R. C. \& LeKA, K. D. 1993 The morphology of flare phenomena, magnetic fields, and electric currents in active regions. III - NOAA active region 6233 (1990 August). Astrophys. J. 411, 378-382.

Del Sarto, D., Pucci, F., Tenerani, A. \& Velli, M. 2016 'Ideal' tearing and the transition to fast reconnection in the weakly collisional MHD and EMHD regimes. J. Geophys. Res. 121, $1857-1873$.

Del Zanna, L., Landi, S., Papini, E., Pucci, F. \& Velli, M. 2016 The ideal tearing mode: theory and resistive MHD simulations. J. Phys.: Conf. Ser. 719 (1), 012016.

Delzanno, G. L. \& Finn, J. M. 2008 The effect of line-tying on tearing modes. Phys. Plasmas 15 (3), 032904.

DÉmoulin, P. 2005 Magnetic Topologies: where Will Reconnection Occur? In Chromospheric and Coronal Magnetic Fields (ed. D. E. Innes, A. Lagg \& S. A. Solanki), ESA Special Publication, vol. 596, p. 22.1.

Démoulin, P., Bagala, L. G., Mandrini, C. H., Hénoux, J. C. \& Rovira, M. G. 1997 Quasi-separatrix layers in solar flares. II. Observed magnetic configurations. Astron. Astrophys. 325, 305-317.

Démoulin, P., HénouX, J. C. \& Mandrini, C. H. 1994 a Are magnetic null points important in solar flares? Astron. Astrophys. 285, p. 1023.

Démoulin, P., HénouX, J. C., Priest, E. R. \& Mandrini, C. H. $1996 a$ Quasi-separatrix layers in solar flares. I. Method. Astron. Astrophys. 308, 643-655.

Démoulin, P., Mandrini, C. H., Rovira, M. G., HénouX, J. C. \& Machado, M. E. $1994 b$ Interpretation of multiwavelength observations of November 5, 1980 solar flares by the magnetic topology of AR 2766. Solar Phys. 150, 221-243.

Démoulin, P., Priest, E. R. \& Lonie, D. P. $1996 b$ Three-dimensional magnetic reconnection without null points 2. Application to twisted flux tubes. J. Geophys. Res. 101, 7631-7646.

Démoulin, P., van Driel-Gesztelyi, L., Schmieder, B., Hénoux, J. C., Csepura, G. \& HAGYARD, M. J. 1993 Evidence for magnetic reconnection in solar flares. Astron. Astrophys. 271, 292.

Drake, J. F., Shay, M. A. \& Swisdak, M. 2008 The Hall fields and fast magnetic reconnection. Phys. Plasmas 15 (4), 042306.

Dudík, J., Janvier, M., Aulanier, G., Del Zanna, G., KarlickÝ, M., Mason, H. E. \& SCHMIEDER, B. 2014 Slipping magnetic reconnection during an X-class solar flare observed by SDO/AIA. Astrophys. J. 784, 144.

Dudík, J., Polito, V., Janvier, M., Mulay, S. M., KarlickÝ, M., Aulanier, G., Del Zanna, G., DzifČÁKová, E., MAson, H. E. \& Schmieder, B. 2016 Slipping magnetic reconnection, chromospheric evaporation, implosion, and precursors in the 2014 September 10 X1.6-Class Solar Flare. Astrophys. J. 823, 41.

Dungey, J. W. 1953 The motion of magnetic fields. Mon. Not. R. Astron. Soc. 113, 679.

Edmondson, J. K., Antiochos, S. K., DeVore, C. R. \& Zurbuchen, T. H. 2010 Formation and reconnection of three-dimensional current sheets in the solar corona. Astrophys. J. 718, $72-85$.

Effenberger, F., Thust, K., Arnold, L., Grauer, R. \& Dreher, J. 2011 Numerical simulation of current sheet formation in a quasiseparatrix layer using adaptive mesh refinement. Phys. Plasmas 18 (3), 032902.

Emslie, A. G., Dennis, B. R., Holman, G. D. \& Hudson, H. S. 2005 Refinements to flare energy estimates: a followup to 'Energy partition in two solar flare/CME events' by A. G. Emslie et al. J. Geophys. Res. 110, A11103.

Feng, L., Wang, Y., Shen, F., Shen, C., Inhester, B., Lu, L. \& Gan, W. 2015 Why does the apparent mass of a coronal mass ejection increase? Astrophys. J. 812, 70.

Fermo, R. L., Drake, J. F. \& Swisdak, M. 2010 A statistical model of magnetic islands in a current layer. Phys. Plasmas 17 (1), 010702. 
Forbes, T. 2000 Solar Flare Models. p. 2295. Institute of Physics Publishing.

Forbes, T. G., Priest, E. R., Seaton, D. B. \& Litvinenko, Y. E. 2013 Indeterminacy and instability in Petschek reconnection. Phys. Plasmas 20 (5), 052902.

Friedman, M. \& Hamberger, S. M. 1968 On the neutral-point region in Petschek's model of magnetic-field annihilation. Astrophys. J. 152, 667.

Furth, H. P., Killeen, J. \& Rosenbluth, M. N. 1963 Finite-resistivity instabilities of a sheet pinch. Phys. Fluids 6, 459-484.

Fuselier, S. A., Lewis, W. S., Schiff, C., Ergun, R., Burch, J. L., Petrinec, S. M. \& TrATtNER, K. J. 2016 Magnetospheric multiscale science mission profile and operations. Space Sci. Rev. 199, 77-103.

GalsgaARD, K. \& Pontin, D. I. 2011 Steady state reconnection at a single 3D magnetic null point. Astron. Astrophys. 529, A20.

GeKelman, W., Lawrence, E. \& Van Compernolle, B. 2012 Three-dimensional reconnection involving magnetic flux ropes. Astrophys. J. 753, 131.

GiBSON, S. E. \& FAN, Y. 2006 Coronal prominence structure and dynamics: a magnetic flux rope interpretation. J. Geophys. Res. 111, A12103.

Gibson, S. E., Kucera, T. A., Rastawicki, D., Dove, J., De Toma, G., Hao, J., Hill, S., Hudson, H. S., Marqué, C. \& McIntosh, P. S. 2010 Three-dimensional morphology of a coronal prominence cavity. Astrophys. J. 724, 1133-1146.

Gilchrist, S. A.\& Wheatland, M. S. 2013 A magnetostatic grad-rubin code for coronal magnetic field extrapolations. Solar Phys. 282, 283-302.

GiovanelLI, R. G. 1947 Magnetic and electric phenomena in the Sun's atmosphere associated with sunspots. Mon. Not. R. Astron. Soc. 107, 338.

Gold, T. \& Hoyle, F. 1960 On the origin of solar flares. Mon. Not. R. Astron. Soc. 120, 89.

Gonzalez, W. \& PARKer, E. (Eds) 2016 Magnetic Reconnection, Astrophysics and Space Science Library, vol. 427.

Gorbachev, V. S. \& Somov, B. V. 1988 Photospheric vortex flows as a cause for two-ribbon flares - a topological model. Solar Phys. 117, 77-88.

Gosling, J. T., Mccomas, D. J., Phillips, J. L. \& BAme, S. J. 1991 Geomagnetic activity associated with earth passage of interplanetary shock disturbances and coronal mass ejections. J. Geophys. Res. 96, 7831-7839.

Graham, D. R. \& CAUZzI, G. 2015 Temporal evolution of multiple evaporating ribbon sources in a solar flare. Astrophys. J. Lett. 807, L22.

Green, L. M. \& Kliem, B. 2009 Flux rope formation preceding coronal mass ejection onset. Astrophys. J. Lett. 700, L83-L87.

Green, L. M., Kliem, B., TöröK, T., van Driel-Gesztelyi, L. \& Attrill, G. D. R. 2007 Transient coronal sigmoids and rotating erupting flux ropes. Solar Phys. 246, 365-391.

Green, R. M. \& SweEt, P. A. 1967 Note on the dissipation of magnetic fields at neutral lines. Astrophys. J. 147, 1153.

HAGYARD, M. J. 1988 Observed nonpotential magnetic fields and the inferred flow of electric currents at a location of repeated flaring. Solar Phys. 115, 107-124.

Hahm, T. S. \& Kulsrud, R. M. 1985 Forced magnetic reconnection. Phys. Fluids 28, 2412-2418.

HAYASHI, T. 1981 Numerical simulations of forced coalescence of magnetic islands generated by a tearing mode instability. J. Phys. Soc. Japan 50, 3124.

Hazeltine, R. D., Meiss, J. D. \& Morrison, P. J. 1986 Analytic theory of the nonlinear $m=1$ tearing mode. Phys. Fluids 29, 1633-1639.

Heerikhuisen, J. \& Craig, I. J. D. 2004 Magnetic reconnection in three dimensions - spine, fan and separator solutions. Solar Phys. 222 (1), 95-114.

Hénoux, J. C., Demoulin, P., Mandrini, C. H. \& Rovira, M. G. 1993 Observational evidence for magnetic reconnection in solar flares (invited). In IAU Colloq. 141: The Magnetic and Velocity Fields of Solar Active Regions (ed. H. Zirin, G. Ai \& H. Wang), Astronomical Society of the Pacific Conference Series, vol. 46, p. 333.

HÉnoux, J. C. \& Somov, B. V. 1987 Generation and structure of the electric currents in a flaring activity complex. Astron. Astrophys. 185, 306-314.

Hesse, M., Forbes, T. G. \& BiRN, J. 2005 On the relation between reconnected magnetic flux and parallel electric fields in the solar corona. Astrophys. J. 631, 1227-1238. 
Hesse, M., Kuznetsova, M. \& Birn, J. 2004 The role of electron heat flux in guide-field magnetic reconnection. Phys. Plasmas 11, 5387-5397.

Hesse, M. \& SCHindleR, K. 1988 A theoretical foundation of general magnetic reconnection. J. Geophys. Res. 93, 5559-5567.

Hirayama, T. 1974 Theoretical model of flares and prominences. I: evaporating flare model. Solar Phys. 34, 323-338.

Hofmann, A., Grigor'ev, V. M. \& Selivanov, V. L. 1988 Analysis and results of cooperative magnetographic measurements. II - comparison and interpretation of the current densities. Astron. Nachr. 309, 373-379.

Hood, A. W. \& PRIEST, E. R. 1979 Kink instability of solar coronal loops as the cause of solar flares. Solar Phys. 64, 303-321.

Hu, Q., Qiu, J., Dasgupta, B., Khare, A. \& Webb, G. M. 2014 Structures of interplanetary magnetic flux ropes and comparison with their solar sources. Astrophys. J. 793, 53.

HubA, J. D. 2003 Hall Magnetohydrodynamics - A Tutorial, pp. 166-192. Springer.

Ishi, Y., AzUmi, M. \& Kishimoto, Y. 2002 Structure-driven nonlinear instability of double tearing modes and the abrupt growth after long-time-scale evolution. Phys. Rev. Lett. 89 (20), 205002.

JAGGI, R. K. 1963 The role of plasma instabilities in the origin of solar flares. J. Geophys. Res. 68, 4429.

Janvier, M., Aulanier, G., Bommier, V., Schmieder, B., Démoulin, P. \& Pariat, E. $2014 a$ Electric currents in flare ribbons: observations and three-dimensional standard model. Astrophys. J. 788, 60 .

Janvier, M., Aulanier, G., Pariat, E. \& Démoulin, P. 2013 The standard flare model in three dimensions. III. Slip-running reconnection properties. Astron. Astrophys. 555, A77.

JANVIER, M., DÉmoulin, P. \& DASSO, S. 2014b Are there different populations of flux ropes in the solar wind? Solar Phys. 289, 2633-2652.

JANVIER, M., Kishimoto, Y. \& LI, J. Q. 2011 Structure-driven nonlinear instability as the origin of the explosive reconnection dynamics in resistive double tearing modes. Phys. Rev. Lett. 107 (19), 195001.

Janvier, M., Savcheva, A., Pariat, E., Tassev, S., Millholland, S., Bommier, V., McCauley, P., McKillop, S. \& Dougan, F. 2016 Evolution of flare ribbons, electric currents, and quasi-separatrix layers during an X-class flare. Astron. Astrophys. 591, A141.

KARlickÝ, M., FÁRník, F. \& MÉszÁrosovÁ, H. 2002 High-frequency slowly drifting structures in solar flares. Astron. Astrophys. 395, 677-683.

Kerr, G. S., Fletcher, L., Russell, A. J. B. \& Allred, J. C. 2016 Simulations of the Mg II k and Ca II 8542 lines from an AlfvÉn Wave-heated Flare Chromosphere. Astrophys. J. 827, 101.

KLIEM, B. 1995 Coupled Magnetohydrodynamic and Kinetic Development of Current Sheets in the Solar Corona, pp. 93-114. Springer.

Kliem, B., Su, Y. N., van Ballegooijen, A. A. \& Deluca, E. E. 2013 Magnetohydrodynamic modeling of the solar eruption on 2010 April 8. Astrophys. J. 779, 129.

Kopp, R. A. \& Pneuman, G. W. 1976 Magnetic reconnection in the corona and the loop prominence phenomenon. Solar Phys. 50, 85-98.

Kosovichev, A. G. \& ZHARkova, V. V. 1999 Variations of photospheric magnetic field associated with flares and CMEs. Solar Phys. 190, 459-466.

Kretzschmar, M. 2011 The Sun as a star: observations of white-light flares. Astron. Astrophys. 530, A84.

Kulsrud, R. M. 2001 Magnetic reconnection: Sweet-Parker versus Petschek. Earth Planets Space 53, 417-422.

Kusano, K., Bamba, Y., Yamamoto, T. T., Iida, Y., Toriumi, S. \& Asai, A. 2012 Magnetic field structures triggering solar flares and coronal mass ejections. Astrophys. J. 760, 31.

LA RosA, T. N. 1990 Impulsive phase heating by uni-directional current systems in solar flares. Solar Phys. 126 (1), 153-175.

Landi, S., Del Zanna, L., Papini, E., Pucci, F. \& Velli, M. 2015 Resistive magnetohydrodynamics simulations of the ideal tearing mode. Astrophys. J. 806, 131.

LAU, Y.-T. \& FINN, J. M. 1990 Three-dimensional kinematic reconnection in the presence of field nulls and closed field lines. Astrophys. J. 350, 672-691. 
LaZarian, A. \& Vishniac, E. T. 1999 Reconnection in a weakly stochastic field. Astrophys. J. 517, 700-718.

Leake, J. E., Lukin, V. S. \& Linton, M. G. 2013 Magnetic reconnection in a weakly ionized plasma. Phys. Plasmas 20 (6), 061202.

Lemen, J. R., Title, A. M., Akin, D. J., Boerner, P. F., Chou, C., Drake, J. F., Duncan, D. W., Edwards, C. G., Friedlaender, F. M., Heyman, G. F. et al. 2012 The atmospheric imaging assembly (AIA) on the solar dynamics observatory (SDO). Solar Phys. 275, $17-40$.

LI, T., YANG, K., Hou, Y. \& Zhang, J. 2016 Slipping magnetic reconnection of flux rope structures as a precursor to an eruptive $\mathrm{X}$-class solar flare. Preprint.

LI, T. \& ZHANG, J. 2014 Slipping magnetic reconnection triggering a solar eruption of a triangleshaped flag flux rope. Astrophys. J. Lett. 791, L13.

Liang, Y., Koslowski, H. R., Krämer-Flecken, A., Zimmermann, O., LöWenbrück, K., Bertschinger, G., WOLF, R. C. \& TEXTOR-TEAM 2007 LETTER: observations of secondary structures after collapse events occurring at the $q=2$ magnetic surface in the TEXTOR tokamak. Nucl. Fusion 47, L21-L25.

Lin, J. \& Forbes, T. G. 2000 Effects of reconnection on the coronal mass ejection process. J. Geophys. Res. 105, 2375-2392.

Liu, W., Chen, Q. \& Petrosian, V. 2013 Plasmoid ejections and loop contractions in an eruptive M7.7 solar flare: evidence of particle acceleration and heating in magnetic reconnection outflows. Astrophys. J. 767, 168.

LiU, W., Jiang, Y. W., LiU, S. \& Petrosian, V. 2004 RHESSI observations of a simple large X-ray flare on 2003 November 3. Astrophys. J. Lett. 611, L53-L56.

LONGCOPE, D. W. 2005 Topological methods for the analysis of solar magnetic fields. Living Rev. Solar Phys. 2, 7.

LongCope, D. W. \& Cowley, S. C. 1996 Current sheet formation along three-dimensional magnetic separators. Phys. Plasmas 3, 2885-2897.

Loureiro, N. F., Cowley, S. C., Dorland, W. D., Haines, M. G. \& Schekochinin, A. A. 2005 X-point collapse and saturation in the nonlinear tearing mode reconnection. Phys. Rev. Lett. 95 (23), 235003.

Loureiro, N. F., Samtaney, R., Schekochinin, A. A. \& Uzdensky, D. A. 2012 Magnetic reconnection and stochastic plasmoid chains in high-Lundquist-number plasmas. Phys. Plasmas 19 (4), 042303.

Loureiro, N. F. \& Uzdensky, D. A. 2016 Magnetic reconnection: from the Sweet-Parker model to stochastic plasmoid chains. Plasma Phys. Control. Fusion 58 (1), 014021.

Lynch, B. J., Antiochos, S. K., DeVore, C. R., Luhmann, J. G. \& Zurbuchen, T. H. 2008 Topological evolution of a fast magnetic breakout CME in three dimensions. Astrophys. $J$. 683, 1192-1206.

Lynch, B. J., Edmondson, J. K., Kazachenko, M. D. \& Guidoni, S. E. 2016 Reconnection properties of large-scale current sheets during coronal mass ejection eruptions. Astrophys. J. 826, 43.

Ma, Z. W. \& Bhattacharjee, A. 1996 Role of photospheric footpoint shear in the impulsive dynamics of the solar corona. Geophys. Res. Lett. 23, 2955-2958.

Mandrini, C. H., Demoulin, P., HenouX, J. C. \& Machado, M. E. 1991 Evidence for the interaction of large scale magnetic structures in solar flares. Astron. Astrophys. 250, 541-547.

Mandrini, C. H., Demoulin, P., Schmieder, B., Deluca, E. E., Pariat, E. \& Uddin, W. 2006 Companion Event and Precursor of the X17 Flare on 28 October 2003. Solar Phys. 238, 293-312.

Mandrini, C. H., Pohjolainen, S., Dasso, S., Green, L. M., Démoulin, P., van Driel-Gesztelyi, L., Copperwheat, C. \& Foley, C. 2005 Interplanetary flux rope ejected from an X-ray bright point. The smallest magnetic cloud source-region ever observed. Astron. Astrophys. 434, 725-740.

Mandrini, C. H., Rovira, M. G., Demoulin, P., Henoux, J. C., Machado, M. E. \& WiLKINSON, L. K. 1993 Evidence for magnetic reconnection in large-scale magnetic structures in solar flares. Astron. Astrophys. 272, 609. 
Masson, S., Pariat, E., Aulanier, G. \& Schrijver, C. J. 2009 The nature of flare ribbons in coronal null-point topology. Astrophys. J. 700, 559-578.

Masuda, S., Kosugi, T., Hara, H., Tsuneta, S. \& Ogawara, Y. 1994 A loop-top hard X-ray source in a compact solar flare as evidence for magnetic reconnection. Nature 371, 495-497.

McKenzie, D. E. 2011 Observational studies of reconnection in the solar corona. Phys. Plasmas 18 (11), 111205.

McKenzie, D. E. \& CAnfield, R. C. 2008 Hinode XRT observations of a long-lasting coronal sigmoid. Astron. Astrophys. 481, L65-L68.

Miklenic, C., Veronig, A. M., Temmer, M., Möstl, C. \& Biernat, H. K. 2011 Coronal dimmings and the early phase of a CME observed with STEREO and Hinode/EIS. Solar Phys. 273, 125-142.

Militello, F., Grasso, D. \& Borgogno, D. 2014 The deceiving $\Delta^{\prime}$ : on the equilibrium dependent dynamics of nonlinear magnetic islands. Phys. Plasmas 21 (10), 102514.

Moreton, G. E. \& Severny, A. B. 1968 Magnetic fields and flares in the region CMP 20 September 1963. Solar Phys. 3, 282-297.

Musset, S., Vilmer, N. \& Bommier, V. 2015 Hard X-ray emitting energetic electrons and photospheric electric currents. Astron. Astrophys. 580, A106.

Ni, L., Germaschewski, K., Huang, Y.-M., Sullivan, B. P., Yang, H. \& Bhattacharjee, A. 2010 Linear plasmoid instability of thin current sheets with shear flow. Phys. Plasmas 17 (5), 052109.

Nindos, A., Patsourakos, S., Vourlidas, A. \& Tagikas, C. 2015 How common are hot magnetic flux ropes in the low solar corona? A statistical study of EUV observations. Astrophys. J. 808, 117.

NishidA, K., Nishizuka, N. \& ShibatA, K. 2013 The role of a flux rope ejection in a threedimensional magnetohydrodynamic simulation of a solar flare. Astrophys. J. Lett. 775, L39.

Nishizuka, N., KARLiCKÝ, M., JANVIER, M. \& BÁRTA, M. 2015 Particle acceleration in plasmoid ejections derived from radio drifting pulsating structures. Astrophys. J. 799, 126.

Pariat, E., Aulanier, G. \& Démoulin, P. 2006 A new concept for magnetic reconnection: sliprunning reconnection. In SF2A-2006: Semaine de l'Astrophysique Francaise (ed. D. Barret, F. Casoli, G. Lagache, A. Lecavelier \& L. Pagani), p. 559.

PARiat, E. \& DÉmoulin, P. 2012 Estimation of the squashing degree within a three-dimensional domain. Astron. Astrophys. 541, A78.

PARKer, E. N. 1957 a Acceleration of cosmic rays in solar flares. Phys. Rev. 107, 830-836.

PARKER, E. N. $1957 b$ Sweet's mechanism for merging magnetic fields in conducting fluids. J. Geophys. Res. 62, 509-520.

PARKER, E. N. 1963 The solar-flare phenomenon and the theory of reconnection and annihiliation of magnetic fields. Astrophys. J. Suppl. 8, 177.

PARKer, E. N. \& Krook, M. 1956 Diffusion and severing of magnetic lines of force. Astrophys. J. 124, 214.

Parnell, C. E., Haynes, A. L. \& GalsgaArd, K. 2010 Structure of magnetic separators and separator reconnection. J. Geophys. Res. 115, 2102.

Parnell, C. E., Smith, J. M., Neukirch, T. \& Priest, E. R. 1996 The structure of threedimensional magnetic neutral points. Phys. Plasmas 3, 759-770.

Petrie, G. J. D. 2013 A spatio-temporal description of the abrupt changes in the photospheric magnetic and Lorentz-force vectors during the 15 February 2011 X2.2 flare. Solar Phys. 287, $415-440$.

Petrie, G. J. D. \& Sudol, J. J. 2010 Abrupt longitudinal magnetic field changes in flaring active regions. Astrophys. J. 724, 1218-1237.

PetscheK, H. E. 1964 Magnetic field annihilation. NASA Special Publication 50, 425.

Petschek, H. E. \& Thorne, R. M. 1967 The existence of intermediate waves in neutral sheets. Astrophys. J. 147, 1157.

Piddington, J. H. $1954 a$ Electromagnetic field equations for a moving medium with Hall conductivity. Mon. Not. R. Astron. Soc. 114, 638.

Piddington, J. H. $1954 b$ The motion of ionized gas in combined magnetic, electric and mechanical fields of force. Mon. Not. R. Astron. Soc. 114, 651. 
Pontin, D. I. 2012 Theory of magnetic reconnection in solar and astrophysical plasmas. Phil. Trans. R. Soc. Lond. A 370, 3169-3192.

Pontin, D. I., Bhattacharjee, A. \& GalsgaArd, K. 2007 Current sheet formation and nonideal behavior at three-dimensional magnetic null points. Phys. Plasmas 14 (5), 052106.

Pontin, D. I. \& CRAIG, I. J. D. 2006 Dynamic three-dimensional reconnection in a separator geometry with two null points. Astrophys. J. 642 (1), 568.

Pontin, D. I., Hornig, G. \& PRIEST, E. R. 2004 Kinematic reconnection at a magnetic null point: spine-aligned current. Geophys. Astrophys. Fluid Dyn. 98, 407-428.

Pontin, D. I., Hornig, G. \& Priest, E. R. 2005 Kinematic reconnection at a magnetic null point: fan-aligned current. Geophys. Astrophys. Fluid Dyn. 99, 77-93.

Pontin, D. I., Priest, E. R. \& GAlsgaARD, K. 2013 On the nature of reconnection at a solar coronal null point above a separatrix dome. Astrophys. J. 774, 154.

Porcelli, F., Borgogno, D., Califano, F., Grasso, D., Ottaviani, M. \& Pegoraro, F. 2002 Recent advances in collisionless magnetic reconnection. Plasma Phys. Control. Fusion 44, 389-405.

Prangé, R., Pallier, L., Hansen, K. C., Howard, R., Vourlidas, A., Courtin, R. \& PARKINSON, C. 2004 An interplanetary shock traced by planetary auroral storms from the Sun to Saturn. Nature 432, 78-81.

PRIEST, E. 2016 MHD structures in three-dimensional reconnection. In Astrophysics and Space Science Library (ed. W. Gonzalez \& E. Parker), Astrophysics and Space Science Library, vol. 427 , p. 101.

Priest, E. R. \& DÉmoulin, P. 1995 Three-dimensional magnetic reconnection without null points. 1. Basic theory of magnetic flipping. J. Geophys. Res. 100, 23443-23464.

PRIEST, E. R. \& Forbes, T. G. 1992 Magnetic flipping - reconnection in three dimensions without null points. J. Geophys. Res. 97, 1521-1531.

Priest, E. R., Hornig, G. \& Pontin, D. I. 2003 On the nature of three-dimensional magnetic reconnection. J. Geophys. Res. 108, 1285.

Priest, E. R. \& Pontin, D. I. 2009 Three-dimensional null point reconnection regimes. Phys. Plasmas 16 (12), 122101.

Priest, E. R. \& RAAdU, M. A. 1975 Preflare current sheets in the solar atmosphere. Solar Phys. 43, 177-188.

Priest, E. R. \& Titov, V. S. 1996 Magnetic reconnection at three-dimensional null points. Proc. R. Soc. Lond. A 354, 2951-2992.

Pritchett, P. L., Lee, Y. C. \& Drake, J. F. 1980 Linear analysis of the double-tearing mode. Phys. Fluids 23, 1368-1374.

PuCcI, F. \& Velli, M. 2014 Reconnection of quasi-singular current sheets: the 'ideal' tearing mode. Astrophys. J. Lett. 780, L19.

QIU, J., HU, Q., Howard, T. A. \& Yurchyshyn, V. B. 2007 On the magnetic flux budget in low-corona magnetic reconnection and interplanetary coronal mass ejections. Astrophys. J. 659, $758-772$.

Reeves, K. K., Linker, J. A., Mikić, Z. \& Forbes, T. G. 2010 Current sheet energetics, flare emissions, and energy partition in a simulated solar eruption. Astrophys. J. 721, 1547-1558.

Restante, A. L., Aulanier, G. \& Parnell, C. E. 2009 How skeletons turn into quasi-separatrix layers in source models. Astron. Astrophys. 508, 433-443.

Rosenbluth, M. N., Dagazian, R. Y. \& Rutherford, P. H. 1973 Nonlinear properties of the internal $m=1$ kink instability in the cylindrical tokamak. Phys. Fluids 16, 1894-1902.

SAKURAI, T. \& UCHIDA, Y. 1977 Magnetic field and current sheets in the corona above active regions. Solar Phys. 52, 397-416.

Samtaney, R., Loureiro, N. F., Uzdensky, D. A., Schekochihin, A. A. \& Cowley, S. C. 2009 Formation of plasmoid chains in magnetic reconnection. Phys. Rev. Lett. 103 (10), 105004.

Savage, S. L., Holman, G., Reeves, K. K., Seaton, D. B., McKenzie, D. E. \& Su, Y. $2012 a$ Low-altitude reconnection inflow-outflow observations during a 2010 November 3 solar eruption. Astrophys. J. 754, 13.

Savage, S. L., McKenzie, D. E. \& Reeves, K. K. $2012 b$ Re-interpretation of supra-arcade downflows in solar flares. Astrophys. J. Lett. 747, L40. 
Savcheva, A., Pariat, E., McKillop, S., McCauley, P., Hanson, E., Su, Y. \& Deluca, E. E. 2016 The relation between solar eruption topologies and observed flare features. II. Dynamical evolution. Astrophys. J. 817, 43.

Savcheva, A., Pariat, E., McKillop, S., McCauley, P., Hanson, E., Su, Y., Werner, E. \& DELUCA, E. E. 2015 The relation between solar eruption topologies and observed flare features. I. Flare Ribbons. Astrophys. J. 810, 96.

Savcheva, A., Pariat, E., van Ballegooijen, A., Aulanier, G. \& Deluca, E. $2012 a$ Sigmoidal active region on the sun: comparison of a magnetohydrodynamical simulation and a nonlinear force-free field model. Astrophys. J. 750, 15.

Savcheva, A. \& VAn BALlegooijen, A. 2009 Nonlinear force-free modeling of a long-lasting coronal sigmoid. Astrophys. J. 703, 1766-1777.

Savcheva, A. S., Green, L. M., van Ballegooijen, A. A. \& Deluca, E. E. $2012 b$ Photospheric flux cancellation and the build-up of sigmoidal flux ropes on the sun. Astrophys. J. 759, 105.

Savcheva, A. S., MCkillop, S. C., McCauley, P. I., Hanson, E. M. \& Deluca, E. E. 2014 A new sigmoid catalog from hinode and the solar dynamics observatory: statistical properties and evolutionary histories. Solar Phys. 289, 3297-3311.

SCHINDler, K., Hesse, M. \& BIRN, J. 1988 General magnetic reconnection, parallel electric fields, and helicity. J. Geophys. Res. 93, 5547-5557.

SCHMidT, H. U. 1964 On the observable effects of magnetic energy storage and release connected with solar flares. NASA Special Publication 50, 107.

Schmieder, B., Aulanier, G., Demoulin, P., Van Driel-Gesztelyi, L., Roudier, T., NitTA, N. \& CAUZZI, G. 1997 Magnetic reconnection driven by emergence of sheared magnetic field. Astron. Astrophys. 325, 1213-1225.

Schmieder, B., Démoulin, P. \& Aulanier, G. 2013 Solar filament eruptions and their physical role in triggering coronal mass ejections. Adv. Space Res. 51, 1967-1980.

Schou, J., Scherrer, P. H., Bush, R. I., Wachter, R., Couvidat, S., Rabello-Soares, M. C., Bogart, R. S., Hoeksema, J. T., LiU, Y., Duvall, T. L. et al. 2012 Design and ground calibration of the helioseismic and magnetic imager (HMI) instrument on the solar dynamics observatory (SDO). Solar Phys. 275, 229-259.

Schrijver, C. J., Beer, J., Baltensperger, U., Cliver, E. W., Güdel, M., Hudson, H. S., Mccracken, K. G., Osten, R. A., Peter, T., Soderblom, D. R. et al. 2012 Estimating the frequency of extremely energetic solar events, based on solar, stellar, lunar, and terrestrial records. J. Geophys. Res. 117, 8103.

Sergeev, V., Kubyshina, M., Alexeev, I., Fazakerley, A., Owen, C., Baumjohann, W., NAKAmura, R., Runov, A., VöRöS, Z., ZHAng, T. L. et al. 2008 Study of near-Earth reconnection events with Cluster and Double Star. J. Geophys. Res. 113, A07S36.

SeVerny, A. 1964a Solar magnetic fields. Space Sci. Rev. 3, 451-486.

Severny, A. B. $1964 b$ Solar flares. Annu. Rev. Astron. Astrophys. 2, 363.

Shay, M. A., Drake, J. F., Swisdak, M. \& Rogers, B. N. 2004 The scaling of embedded collisionless reconnection. Phys. Plasmas 11, 2199-2213.

Shibata, K. \& TANuma, S. 2001 Plasmoid-induced-reconnection and fractal reconnection. Earth Planets Space 53, 473-482.

Sobotka, M., Dudík, J., Denker, C., Balthasar, H., Jurčák, J., Liu, W., Berkefeld, T., Collados Vera, M., Feller, A., Hofmann, A. et al. 2016 Slipping reconnection in a solar flare observed in high resolution with the GREGOR solar telescope. Preprint.

Sonnerup, B. U. Ö. 1970 Magnetic-field re-connexion in a highly conducting incompressible fluid. J. Plasma Phys. 4, 161-174.

SPICER, D. S. 1981 First phase heating and particle acceleration during solar flares by fast tearing modes. Solar Phys. 71, 115-124.

SPITZER, L. 1956 Physics of Fully Ionized Gases.

Stenzel, R. L., Urrutia, J. M., Griskey, M. \& Strohmaier, K. 2002 A new laboratory experiment on magnetic reconnection. Phys. Plasmas 9, 1925-1930.

Stevenson, J. E. H. \& PARnell, C. E. 2015 Spontaneous reconnection at a separator current layer: 1. Nature of the reconnection. J. Geophys. Res. 120, 10.

Sturrock, P. A. 1966 Model of the high-energy phase of solar flares. Nature 211, 695-697. 
Sturrock, P. A. 1968 A model of solar flares. In Structure and Development of Solar Active Regions (ed. K. O. Kiepenheuer), IAU Symposium, vol. 35, p. 471.

Su, Y., Golub, L., van Ballegooijen, A., Deluca, E. E., Reeves, K. K., Sakao, T., Kano, R., NARUKage, N. \& Shibasaki, K. 2007 Evolution of the sheared magnetic fields of two X-class flares observed by Hinode/XRT. Pub. Astron. Soc. Japan 59, 785.

Su, Y. N., Golub, L., van Ballegooijen, A. A. \& Gros, M. 2006 Analysis of magnetic shear in An X17 solar flare on October 28, 2003. Solar Phys. 236, 325-349.

Sudol, J. J. \& HARVEY, J. W. 2005 Longitudinal magnetic field changes accompanying solar flares. Astrophys. J. 635, 647-658.

Sun, X., Hoeksema, J. T., Liu, Y., Wiegelmann, T., Hayashi, K., Chen, Q. \& Thalmann, J. 2012 Evolution of magnetic field and energy in a major eruptive active region based on SDO/HMI observation. Astrophys. J. 748, 77.

SVESTKA, Z. 1986 On the varieties of solar flares. In The Lower Atmosphere of Solar Flares; Proceedings of the Solar Maximum Mission Symposium, Sunspot, NM, Aug. 20-24, 1985 (A87-26201 10-92). Sunspot, NM, National Solar Observatory, 1986, pp. 332-355 (ed. D. F. Neidig), pp. 332-355.

SweEt, P. A. 1950 The effect of turbulence on a magnetic field. Mon. Not. R. Astron. Soc. 110, 69-83.

SWeEt, P. A. 1956 Proceedings of the International Astronomical Union Symposium on Electromagnetic Phenomena in Cosmical Physics, Stockholm, 1956, IAU Symposium; unpublished.

Sweet, P. A. $1958 a$ The neutral point theory of solar flares. In Electromagnetic Phenomena in Cosmical Physics (ed. B. Lehnert), IAU Symposium, vol. 6, p. 123.

SweET, P. A. $1958 b$ The production of high energy particles in solar flares. In Supplemento al volume VIII, Serie X Del Nuovo Cimento, vol. 8, pp. 188-196.

SweEt, P. A. $1958 c$ The topology of force-free magnetic fields. Observatory 78, 30-32.

Sweet, P. A. 1969 Mechanisms of solar flares. Annu. Rev. Astron. Astrophys. 7, 149.

SYROVATSKII, S. I. 1966 Dynamic dissipation of a magnetic field and particle acceleration. Sov. Astron. 10, 270.

Syrovatskit, S. I., Frank, A. G. \& Khodzhaev, A. Z. 1972 Development of a current layer when plasma moves in a magnetic field with a neutral line. Sov. J. Exp. Theoret. Phys. Lett. $15,94$.

SYROVATSKIǏ, S. I. 1971 Formation of current sheets in a plasma with a frozen-in strong magnetic field. Sov. J. Exp. Theoret. Phys. 33, 933.

Takasao, S., Asai, A., Isobe, H. \& ShibatA, K. 2012 Simultaneous observation of reconnection inflow and outflow associated with the 2010 August 18 solar flare. Astrophys. J. Lett. 745, L6.

TASSEV, S. \& SAVChEVA, A. 2016 QSL squasher: a fast quasi-separatrix layer map calculator. Preprint.

Titov, V. S., Forbes, T. G., Priest, E. R., Mikić, Z. \& Linker, J. A. 2009 Slip-squashing factors as a measure of three-dimensional magnetic reconnection. Astrophys. J. 693, 1029-1044.

Titov, V. S., Hornig, G. \& DÉmoulin, P. 2002 Theory of magnetic connectivity in the solar corona. J. Geophys. Res. 107, 1164.

TÖRÖK, T. \& KLIEM, B. 2005 Confined and ejective eruptions of kink-unstable flux ropes. Astrophys. J. Lett. 630, L97-L100.

Tripathi, D., Isobe, H. \& Mason, H. E. 2006 On the propagation of brightening after filament/prominence eruptions, as seen by SoHO-EIT. Astron. Astrophys. 453, 1111-1116.

Tripathi, D., Kliem, B., Mason, H. E., Young, P. R. \& Green, L. M. 2009 Temperature tomography of a coronal sigmoid supporting the gradual formation of a flux rope. Astrophys. J. Lett. 698, L27-L32.

TSIKlaURI, D. \& HARUKI, T. 2007 Magnetic reconnection during collisionless, stressed, X-point collapse using particle-in-cell simulation. Phys. Plasmas 14 (11), 112905-112905.

TUR, T. J. \& PRIEST, E. R. 1976 The formation of current sheets during the emergence of new magnetic flux from below the photosphere. Solar Phys. 48, 89-100.

Uzdensky, D. A., Loureiro, N. F. \& Schekochinin, A. A. 2010 Fast magnetic reconnection in the plasmoid-dominated regime. Phys. Rev. Lett. 105 (23), 235002. 
VAn Ballegooijen, A. A. 2004 Observations and modeling of a filament on the sun. Astrophys. J. 612, 519-529.

van Hoven, G. \& Cross, M. A. 1973 Energy release by magnetic tearing: the nonlinear limit. Phys. Rev. A 7, 1347-1352.

Vekstein, G., Priest, E. R. \& Amari, T. 1991 Formation of current sheets in force-free magnetic fields. Astron. Astrophys. 243, 492-500.

Waelbroeck, F. L. 1989 Current sheets and nonlinear growth of the $m=1$ kink-tearing mode. Phys. Fluids B 1, 2372-2380.

Wang, H., Spirock, T. J., Qiu, J., Ji, H., Yurchyshyn, V., Moon, Y.-J., Denker, C. \& Goode, P. R. 2002 Rapid changes of magnetic fields associated with six X-class flares. Astrophys. J. 576, 497-504.

Wang, S., LiU, C., LiU, R., Deng, N., LiU, Y. \& WANG, H. 2012 Response of the photospheric magnetic field to the X2.2 flare on 2011 February 15. Astrophys. J. Lett. 745, L17.

Wang, S., Yokoyama, T. \& Isobe, H. 2015 Three-dimensional MHD magnetic reconnection simulations with a finite guide field: proposal of the shock-evoking positive-feedback model. Astrophys. J. 811, 31.

WANG, T., SUI, L. \& QIU, J. 2007 Direct observation of high-speed plasma outflows produced by magnetic reconnection in solar impulsive events. Astrophys. J. Lett. 661 (2), L207.

Wang, X., Ma, Z. W. \& Bhattacharjee, A. 1996 Fast magnetic reconnection and sudden enhancement of current sheets due to inward boundary flows. Phys. Plasmas 3, 2129-2134.

Wang, Z. X., Wang, X. G., Dong, J. Q., Lei, Y. A., Long, Y. X., Mou, Z. Z. \& QU, W. X. 2007 Fast resistive reconnection regime in the nonlinear evolution of double tearing modes. Phys. Rev. Lett. 99 (18), 185004.

Warren, H. P., O'Brien, C. M. \& Sheeley, N. R. JR. 2011 Observations of reconnecting flare loops with the atmospheric imaging assembly. Astrophys. J. 742, 92.

Watanabe, K., Sato, T. \& Nakayama, Y. 1995 Current profile flattening and hot core shift due to non-linear development of resistive kink mode. Nucl. Fusion 35, 251-259.

Webb, D. F. \& Howard, T. A. 2012 Coronal mass ejections: observations. Living Rev. Solar Phys. 9 (3), 3.

Wendel, D. E., Olson, D. K., Hesse, M., Aunai, N., Kuznetsova, M., Karimabadi, H., Daughton, W. \& AdriAn, M. L. 2013 The relation between reconnected flux, the parallel electric field, and the reconnection rate in a three-dimensional kinetic simulation of magnetic reconnection. Phys. Plasmas 20 (12), 122105.

Wentzel, D. G. 1963 Dissipation of magnetic energy in a solar flare. Astronom. J. 68, 299.

Wild, J. P., Smerd, S. F. \& Weiss, A. A. 1963 Solar bursts. Annu. Rev. Astron. Astrophys. 1, 291.

Wilmot-Smith, A. L., Hornig, G. \& Pontin, D. I. 2009 Magnetic braiding and quasi-separatrix layers. Astrophys. J. 704, 1288-1295.

Wyper, P. F., DeVore, C. R., KArpen, J. T. \& LynCh, B. J. 2016 Three-dimensional simulations of tearing and intermittency in coronal jets. Astrophys. J. 827, 4.

Wyper, P. F. \& Pontin, D. I. 2014 Dynamic topology and flux rope evolution during non-linear tearing of 3D null point current sheets. Phys. Plasmas 21 (10), 102102.

YAMADA, M. 2007 Progress in understanding magnetic reconnection in laboratory and space astrophysical plasmas. Phys. Plasmas 14 (5), 058102.

Yamada, M., Ji, H., Hsu, S., Carter, T., Kulsrud, R., Bretz, N., Jobes, F., Ono, Y. \& PERKINS, F. 1997 Study of driven magnetic reconnection in a laboratory plasma. Phys. Plasmas 4, 1936-1944.

Yamada, M., Kulsrud, R. \& Ji, H. 2010 Magnetic reconnection. Rev. Mod. Phys. 82, $603-664$.

Yamada, M., Yoo, J., Jara-Almonte, J., Daughton, W., Ji, H., Kulsrud, R. M. \& MYERS, C. E. 2015 Study of energy conversion and partitioning in the magnetic reconnection layer of a laboratory plasma). Phys. Plasmas 22 (5), 056501.

YAmadA, M., Yoo, J. \& ZENITANi, S. 2016 Energy conversion and inventory of a prototypical magnetic reconnection layer. In Astrophysics and Space Science Library (ed. W. Gonzalez \& E. Parker), Astrophysics and Space Science Library, vol. 427, p. 143. 
Yashiro, S., Akiyama, S., Gopalswamy, N. \& Howard, R. A. 2006 Different power-law indices in the frequency distributions of flares with and without coronal mass ejections. Astrophys. $J$. Lett. 650, L143-L146.

YEH, T. 1976 Diffusive hydromagnetic flow in the vicinity of a neutral point. Astrophys. J. 207, 837-847.

YeH, T. \& AXFORD, W. I. 1970 On the re-connexion of magnetic field lines in conducting fluids. J. Plasma Phys. 4, 207-229.

Yokoyama, T., Akita, K., Morimoto, T., Inoue, K. \& Newmark, J. 2001 Clear evidence of reconnection inflow of a solar flare. Astrophys. J. Lett. 546, L69-L72.

Zhao, J., Gilchrist, S. A., Aulanier, G., Schmieder, B., Pariat, E. \& Li, H. 2016 Hooked flare ribbons and flux-rope-related QSL footprints. Astrophys. J. 823, 62.

Zheng, R., ChEn, Y.\& WANG, B. 2016 Slipping magnetic reconnections with multiple flare ribbons during an X-class solar flare. Astrophys. J. 823, 136.

Zurbuchen, T. H. \& Richardson, I. G. 2006 In-situ solar wind and magnetic field signatures of interplanetary coronal mass ejections. Space Sci. Rev. 123, 31-43.

Zweibel, E. G. 2015 Ambipolar diffusion. In Magnetic Fields in Diffuse Media (ed. A. Lazarian, E. M. de Gouveia Dal Pino \& C. Melioli), Astrophysics and Space Science Library, vol. 407, p. 285.

Zweibel, E. G. \& Yamada, M. 2009 Magnetic reconnection in astrophysical and laboratory plasmas. Annu. Rev. Astron. Astrophys. 47, 291-332.

\section{Miho Janvier}

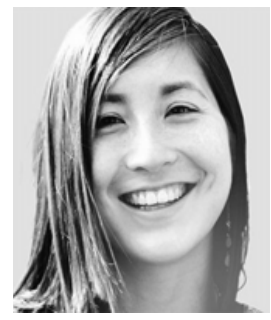

Miho Janvier is an associate astronomer at the Institut d'Astrophysique Spatiale, Université Paris-Sud in France. She obtained her $\mathrm{PhD}$ in plasma physics in 2011 from Kyoto University in Japan, where she worked on numerical simulations of plasma instabilities leading to magnetic reconnection. She obtained an AXA Research Fund Post-doctoral Fellowship to pursue her research in solar and space physics at the Observatoire de Paris, LESIA (France) for two years, before taking up a position as a lecturer at the University of Dundee (UK) from 2013 to 2015.

Her research focusses on the underlying mechanisms of solar flares by analysing both space-borne observations and three-dimensional magnetohydrodynamic numerical simulations. She also works on the development of models derived from the analysis of in situ data to understand the generic shapes of interplanetary coronal mass ejections, a consequence of solar flares. She is currently a member of the operation team for the spectrograph SPICE and a Co-I on the EUV imager EUI, both on the future Solar Orbiter mission. 